\author{
Universidade de São Paulo \\ Instituto de Física \\ Departamènto de Física Matemática
}

\title{
EFEITOS DINÂMICOS DAS FLUTUAÇÕES DOS CAMPOS MAGNÉTICOS DO VÁCUO: RUÍDO RADIATIVO EM CIRCUITOS COM INDUTÂNCIA
}

Alencar José de Faria

Banca Examinadora

Prof. Dr. Humberto de Menezes França (IFUSP) - Orientador Prof. Dr. Antonio Vidiella Barranco (IFGW - UNICAMP) Profa. Dra. Coraci Pereira Malta (IFUSP)

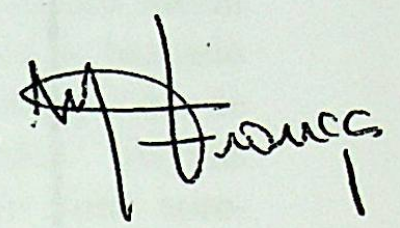

Dissertação apresentada ao Instituto de Física da Universidade de São Paulo para a obtenção do título

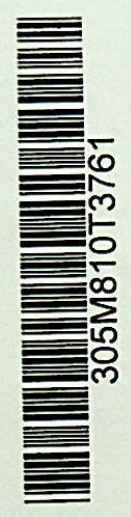
de Mestre em Ciências.

São Paulo

Dezembro de 2002

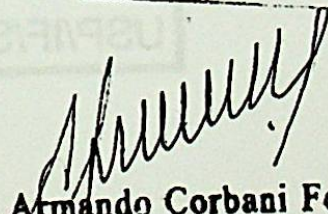
'residente/da Comissão de Pós Graduachar. 


\section{FICHA CATALOGRÁFICA}

Preparada pelo Serviço de Biblioteca e Informação do Instituto de Física da Universidade de São Paulo

Faria, Alencar José de

Efeitos Dinâmicos das Flutuações dos Campos Magnéticos do Vácuo: Ruido Radiativo em Circuitos com Indutância. São Paulo, 2002

Dissertação (Mestrado) Universidade de São Paulo Instituto de Fisica. Departamento de Física Matemática

Orientador: Prof. Dr. Humberto de Menezes França Área de Concentração: Fisica

Unitermos: 1. Ruido em Condutores;

2. Eletrodinâmica Estocástica;

3. Flutuações do Vácuo;

4. Ruido de Nyquist. 


\section{Resumo}

Examinamos os efeitos do campo magnético do vácuo (térmico e de pontozero) sobre um solenóide macroscópico de um circuito elétrico. Concluimos que existem flutuações de tensão e corrente no circuito associadas à ação do campo magnético flutuante nas espiras do solenóide. Este efeito foi denominado ruído radiativo. $O$ primeiro cálculo publicado utiliza o teorema flutuação-dissipação para mostrar a possibilidade de existência do ruído radiativo. A demonstração do ruído radiativo em circuitos com indutância foi feita por nós através de um cálculo detalhado do vetor de Poynting dos campos em torno do solenóide. Apresentamos também diversos efeitos devidos à interação entre o solenóide macroscópico e um dipolo elétrico microscópico, intermediada pelos campos eletromagnéticos do vácuo. Os cálculos foram realizados no âmbito da Eletrodinâmica Estocástica, uma teoria baseada no Eletromagnetismo Clássico, na Mecânica Relativística e na hipótese de existência real do campo eletromagnético de ponto-zero clássico. Alguns desenvolvimentos básicos dessa teoria são apresentados. Além disso apresentamos a descrição detalhada das flutuações térmicas e de ponto-zero da tensão e da corrente em circuitos elétricos, conhecidas como ruído de Nyquist, visando uma melhor compreensão do estudo do ruído radiativo, previsão original desta dissertação. 


\begin{abstract}
We analyze the effects of the vacuum magnetic field (thermal and zero-point) on a macroscopic solenoid of an electric circuit. We conclude that there are voltage and current fluctuations associated to the action of the fluctuating magnetic field on the solenoid coils. This effect was denominated radiative noise. In the first published calculation, the fluctuation-dissipation theorem was used to show the possibility of existence of the radiative noise. The proof of the radiative noise in circuits with inductance has been done by us through the detailed calculation of the Poynting vector around the solenoid. We also present some effects due to the interaction between the macroscopic solenoid and a microscopic electric dipole, intermediated by the vacuum electromagnetic fields. The calculations were performed within the realm of the Stochastic Electrodynamics formalism. This theory is based on the Classical Electromagnetism, the Relativistic Mechanics and the hypothesis of real existence of the classical zero-point electromagnetic field. We show some of the basic results of the Stochastic Electrodynamics approach. Moreover, we introduce a description of the thermal and the zero-point voltage and current fluctuations in electric circuits, the well known Nyquist noise, aiming to achieve a better comprehension of the radiative noise effects, original prediction of this report.
\end{abstract}


À memória de meu pai, José Araripe,

e à minha mãe, Maria Ivete.

Meu filho, não esqueças minha instrução, guarda no coração os meus preceitos.

Provérbios 3,1 


\section{Agradecimentos}

- Nada pode existir no vazio. A conquista desta etapa só foi possível com o apoio que recebi de muitas pessoas. Em primeiro lugar devo agradecer ao Professor Humberto M. França, pelo seu estímulo ao pensamento crítico e pela sua paciência.

- Agradeço profundamente a meu pai e a minha mãe. Agradeço também a meus irmão e parentes próximos.

- Agradeço aos meus amigos com quem convivi no Departamento de Física Matemática, pelas amizades, principalmente, mas também pelas ajudas que me deram. Em especial agradeço ao Marcelo Pires, João Luis, Rodrigo Fresneda, Márcio (Gaúcho), Masayuki, Arnaldo, Mário, Carlos, Leonardo, Fábio, Rodrigo Sponchiado, Samira, José Cleriston, Lamartine e Ronaldo.

- Agradeço também aos meus amigos da graduação, tanto pelas discussões sérias como pelos momentos de descontração. Devo lembrar da Bruna, Cassius, Leo, Sávio, Gustavo, Luciana Ghussn, Nelson, André Sardão, Rodrigo Cuzinatto, Thiago, Jorge, Ciro, Pedro, Caio, Matheus, Éder, Péricles, Marco Antônio, Armando, Maurício Trota, Flávio (Agro) e Itamar. Acompanhando estas pessoas, agradeço também a todos os jaguarianos.

- Agradeço à FAPESP, Fundação de Amparo à Pesquisa do Estado de São Paulo, pelo apoio financeiro. 


\section{Sumário}

1 Introdução

2 Eletrodinâmica Estocástica 8

2.1 Resultados Elementares . . . . . . . . . . . . . 8 8

2.1.1 Radiação de Ponto-Zero . . . . . . . . . . . . . 8

2.1 .2 Radiação de Corpo Negro . . . . . . . . . . . . 12

2.2 Funções de Correlação para Campos Eletromagnéticos Livres . 15

2.3 Oscilador Elétrico Dipolar . . . . . . . . . . . . . 20

3 O Ruído de Nyquist 28

3.1 Equação de Langevin . . . . . . . . . . . . . . 28

3.2 Ruído de Nyquist . . . . . . . . . . . . . . . 33

4 Interação do solenóide com o oscilador dipolar elétrico $\quad 37$

4.1 Descrição do sistema físico . . . . . . . . . . 38

4.2 Flutuações do sistema e equações da interação . . . . . . . 39

4.3 Propriedades do dipolo elétrico . . . . . . . . . . . 42

4.4 Emissão espontânea . . . . . . . . . . . . . . . . . 44

4.5 Densidade espectral das tensões flutuantes no circuito . . . . 46

5 Ruído Radiativo $\quad 47$

5.1 Ruído radiativo em circuitos com indutância . . . . . . . 48

5.2 Demostração da presença do ruído radiativo . . . . . . . 52

5.2 .1 Introdução . . . . . . . . . . . . . . 52

5.2.2 Cálculo dos campos elétrico e magnético gerados pelo solenóide . . . . . . . . . . . . . 53

5.2.3 A média do vetor de Poynting . . . . . . . . 56

5.2.4 Condições para a medição do ruído radiativo . . . . . 59 
A Campos de interação entre o solenóide e o dipolo elétrico 66 A.1 Campo elétrico gerado pelo solenóide . . . . . . . . 66

A.2 Força eletromotriz induzida pelo dipolo elétrico . . . . . . 68

Referências Bibliográficas 


\section{Capítulo 1}

\section{Introdução}

O trabalho teórico nas ciências físicas só pode existir e desenvolver-se através da comparação com a evidência experimental. Não há dúvida de que o sucesso de uma teoria física deve-se ao grau de detalhamento e acordo com os fatos experimentais. Para tal é necessário que a teoria nos dê uma compreensão unificadora dos fenômenos, de modo que seja possível um vínculo entre os conceitos teóricos e os fatos objetivos. A partir desse aspecto, embora a Mecânica Quântica e, consequentemente, a Eletrodinâmica Quântica sejam as teorias físicas mais bem verificadas experimentalmente, as várias interpretações que essas teorias possibilitam bem como suas incongruências com o mundo clássico, mostram que existem alguns elementos obscuros que precisam ser esclarecidos.

Diversos autores fizeram várias tentativas para resolver essas questões, dentre eles podemos citar Einstein, Planck e muitos outros. A partir de alguns desses trabalhos pioneiros, foi desenvolvida em meados do século passado uma nova teoria, chamada de Eletrodinâmica Estocástica, que poderia tornar-se uma opção paralela às teorias quânticas.

No Eletromagnetismo Clássico, as soluções das equações de Maxwell para o vácuo, sem fontes e ondas eletromagnéticas, são ajustadas, por condições de contorno apropriadas, a serem nulas. A Eletrodinâmica Estocástica é constituída pelo Eletromagnetismo Clássico, porém com as condições de contorno tais que as soluções homogêneas das equações de Maxwell nunca são nulas, de modo que sempre haja um campo aleatório chamado de campo eletromagnético de ponto-zero clássico. Para a descrição dos movimentos de partículas, a Eletrodinâmica Estocástica se fundamenta na Mecânica Relativística, além dos outros fundamentos já citados. O acréscimo da hipótese 
do campo de ponto-zero, que deve ter média temporal e espacial nula, tem como objetivo descrever os fenômenos microscópicos probabilísticos, que são usualmente descritos pelas teorias quânticas.

Um dos objetivos principais desta dissertação é mostrar que problemas que são comumente resolvidos pelas teorias quânticas também podem ser resolvidos através de uma formulação clássica adequada, que pode, além disso, oferecer novas contribuições. Assim os sistemas físicos aqui estudados são analisados através do formalismo da Eletrodinâmica Estocástica.

Procurando tornar claros os seus princípios e o motivo do seu uso, alguns resultados fundamentais da Eletrodinâmica Estocástica são apresentados no Capítulo 2. Na primeira seção é feita a dedução da distribuição espectral do campo eletromagnético de ponto-zero, usando apenas o Eletromagnetismo Clássico e a propriedade de invariância de Lorentz. Obtido esse resultado inicial, é apresentada a seguir a demonstração da distribuição espectral da radiação de corpo negro, sem usar hipóteses quânticas, o que nos mostra um dos grandes sucessos da Eletrodinâmica Estocástica. As duas últimas partes do Capítulo 2 são dedicadas à comparação entre a Eletrodinâmica Estocástica e a Eletrodinâmica Quântica para dois sistemas físicos importantíssimos, os campos eletromagnéticos livres e o oscilador elétrico dipolar. A partir dessa comparação é possível estabelecer uma relação geral entre ambas teorias.

O estudo de ruídos em circuitos elétricos lineares é uma das preocupações principais desta dissertação. Em condutores elétricos, em geral, e particularmente em circuitos elétricos, existe a presença de flutuações das tensões e correntes elétricas, mesmo quando estão desligados de qualquer bateria. Este ruído, devido à agitação térmica dos elétrons contidos nos condutores, é chamado de ruído de Nyquist. No Capítulo 3, apresentamos alguns tópicos diretamente relacionados com o ruído de Nyquist. Inicialmente são mostradas a construção e as conseqüências da equação de Langevin de sistemas estocásticos, que no caso são circuitos RLC série com ruído. A seguir é feita uma demonstração da relação de Nyquist, que pode ser generalizada no teorema flutuação-dissipação, o que nos fornece uma relação geral entre as componentes dissipativas e flutuantes de sistemas lineares.

No Capítulo 4 apresentamos desenvolvimentos que foram feitos apenas utilizando o formalismo da Eletrodinâmica Estocástica. Seguindo os trabalhos feitos por Blanco, Dechoum, França, Santos e Maia Jr. [1, 2, 3], são mostrados os efeitos de um oscilador dipolar elétrico microscópico próximo a um solenóide de um circuito RLC. O solenóide e o oscilador dipolar elétrico estão imersos em um banho radiativo a uma certa temperatura. Pelos tra- 
balhos apresentados em $[1,2,3]$, os tempos de vida dos estados excitados do oscilador são modificados pela presença do solenóide, além do ruído presente no circuito também ser modificado pela presença do oscilador microscópico. São descritos como o estado de equilíbrio e os estados excitados do oscilador são afetados por esse arranjo $[1,3]$.

O principal objetivo desta dissertação é, através da Eletrodinâmica Estocástica, obter contribuições novas que possam ser verificadas experimentalmente. Então, foram estudados sistemas físicos mais realistas, de modo que pudessem revelar novos aspectos teóricos, e assim servir à comparação entre a Eletrodinâmica Estocástica, o formalismo quântico e os dados experimentais. Apresentamos no Capítulo 5 dois trabalhos feitos recentemente $[4,5]$, nos quais se estuda um circuito com indutância, constituído por um solenóide que possue dimensões macroscópicas específicas, imerso em um banho radiativo. A análise feita por Blanco, França, Santos e Sponchiado [4] utiliza o teorema flutuação-dissipação para mostrar a existência de um novo ruído presente no circuito, diferente do ruído de Nyquist usual. Essa nova contribuição das correntes e tensões flutuantes é associada à resistência radiativa do solenóide. A energia acumulada pelo circuito RLC também é calculada. Para que o conceito macroscópico de resistência radiativa e as flutuações microscópicas de corrente e tensão dos estudos feitos em [4] fossem esclarecidos, foi realizado um trabalho por este autor, França e Sponchiado [5] no qual se analisa o fluxo de energia entre o solenóide do circuito RLC e os campos flutuantes do vácuo. Esse estudo [5] demonstrou a existência do ruído radiativo, além de verificar que o espectro do campo do vácuo resultante é estável, embora seja modificado nas proximidades do solenóide. 


\section{Capítulo 2}

\section{Eletrodinâmica Estocástica}

\subsection{Resultados Elementares}

A Eletrodinâmica Estocástica (conhecida na literatura como SED) sustentase na Eletrodinâmica Clássica, na Mecânica Clássica Relativística e na hipótese da existência de um campo eletromagnético de ponto-zero, cuja distribuição espectral é isotrópica, espacialmente homogênea e invariante de Lorentz. A hipótese da radiação de ponto-zero respeita as bases teóricas da SED, pois podemos adequar as condições de contorno das equações de Maxwell, de modo que as soluções homogêneas tenham um termo aleatório, cuja média temporal ou espacial é nula. A invariância de Lorentz é uma escolha natural, visto que o espectro da radiação de ponto-zero deve ser o mesmo, independentemente do referencial inercial utilizado. Para mais detalhes sobre a Eletrodinâmica Estocástica, há as referências $[9,13]$. Os leitores já familiarizados com a SED podem prosseguir a leitura desta dissertação a partir do capítulo 3 .

\subsubsection{Radiação de Ponto-Zero}

O primeiro passo a ser dado na SED é determinar a distribuição espectral do campo eletromagnético de ponto-zero. A demonstração apresentada aqui segue um trabalho feito por Boyer [11], em que as componentes elétrica e magnética da radiação de ponto-zero são variáveis estocásticas, tratadas pelo método de Einstein e Hopf [10]. Para simplificação dos cálculos, sem perda de generalidade, as expressões dos campos podem ser escritas de forma que não sejam manifestamente invariantes de Lorentz, logo o uso do gauge de Coulomb é adequado para os cálculos. Assim o campo elétrico livre no vácuo, 
$\vec{E}$, pode ser expandido em uma soma de ondas planas, isto é,

$$
\vec{E}(\vec{r}, t)=\Re \mathfrak{R e} \sum_{\alpha=1}^{2} \int d^{3} k \hat{\epsilon}(\vec{k}, \alpha) \mathfrak{h}(\vec{k}) e^{i \omega t-i \vec{k} \cdot \vec{r}-i \theta(\vec{k}, \alpha)},
$$

o mesmo para o campo magnético $\vec{B}$,

$$
\vec{B}(\vec{r}, t)=\Re e \sum_{\alpha=1}^{2} \int d^{3} k \frac{\vec{k} \times \hat{\epsilon}(\vec{k}, \alpha)}{k} \mathfrak{h}(\vec{k}) e^{i \omega t-i \vec{k} \cdot \vec{r}-i \theta(\vec{k}, \alpha)},
$$

sendo que para cada vetor de onda $\vec{k}$, cujo o módulo é $|\vec{k}|=k=\omega / c$, estão associados dois versores de polarização $\hat{\epsilon}(\vec{k}, \alpha), \alpha \in\{1,2\}$, linearmente independentes. Estes vetores satisfazem as seguintes relações:

$$
\begin{gathered}
\vec{k} \cdot \hat{\epsilon}(\vec{k}, \alpha)=0, \\
\hat{\epsilon}(\vec{k}, \alpha) \cdot \hat{\epsilon}\left(\vec{k}, \alpha^{\prime}\right)=\delta_{\alpha \alpha^{\prime}}, \\
\sum_{\alpha=1}^{2} \epsilon_{i}(\vec{k}, \alpha) \epsilon_{j}(\vec{k}, \alpha)=\delta_{i j}-\frac{k_{i} k_{j}}{k^{2}} .
\end{gathered}
$$

Para cada onda das expansões de $\vec{E}$ e $\vec{B}$, com vetor de onda $\vec{k}$ e polarização $\alpha$, existe uma fase aleatória $\theta(\vec{k}, \alpha)$, cuja distribuição de probabilidades é uniforme e não nula no intervalo $0 \leq \theta<2 \pi$. A função $\mathfrak{h}(\vec{k})$, que representa a amplitude de cada onda plana das expansões (2.1) e (2.2), é a princípio desconhecida. Como a radiação de ponto-zero deve ser homogênea e isotrópica, a função $\mathfrak{h}$ deve depender apenas de $\omega=c k$.

Para que seja possível vincular $\mathfrak{h}$ com a distribuição espectral $\rho(\omega)$, calculase a densidade de energia, isto é,

$$
\frac{1}{8 \pi}\left\langle\vec{E}^{2}+\vec{B}^{2}\right\rangle=\frac{1}{8 \pi} \sum_{\alpha=1}^{2} \int d^{3} k \mathfrak{h}^{2}(\omega)=\int_{0}^{\infty} d \omega \frac{\omega^{2}}{c^{3}} \mathfrak{h}^{2}(\omega),
$$

visto que foi feita a média nas fases aleatórias (veja [11])

$$
\left\langle e^{-i \theta(\vec{k}, \alpha)} e^{i \theta\left(\vec{k}^{\prime}, \alpha^{\prime}\right)}\right\rangle=\delta_{\vec{k} \vec{k}^{\prime}} \delta_{\alpha \alpha^{\prime}} .
$$

Pelo último membro da equação (2.4), a seguinte definição para densidade espectral $\rho(\omega)$ pode ser estabelecida,

$$
\rho(\omega) \equiv \frac{\omega^{2}}{c^{3}} \mathfrak{h}^{2}(\omega)
$$


Aplicando uma transformação de Lorentz neste sistema, as expressões dos campos elétrico e magnético necessariamente mudam, porém, dentro das hipóteses estabelecidas, a distribuição espectral não deve ser alterada. Assim vamos considerar que os cálculos feitos até aqui são para um referencial $\mathrm{S}$, cujos eixos de coordenadas são $0-x, 0-y$ e $0-z$, nas direções $\hat{i}, \hat{j}$ e $\hat{k}$ respectivamente. Um referencial S' move-se com velocidade $\vec{v}=v \hat{i}$ em relação ao referencial S. Vamos escrever as variáveis do referencial S' assim como as variáveis do referencial $\mathrm{S}$ mais o acréscimo de uma linha \%. Os campos elétrico $\vec{E}^{\prime}$ e magnético $\vec{B}^{\prime}$ em $S^{\prime}$ são (vide [16])

$$
\begin{array}{r}
\vec{E}^{\prime}\left(\vec{r}^{\prime}, t^{\prime}\right)=\Re e \sum_{\alpha=1}^{2} \int d^{3} k \mathfrak{h}(\vec{k}) e^{i \omega^{\prime} t^{\prime}-i \vec{k}^{\prime} \cdot \vec{r}^{\prime}-i \theta\left(\vec{k}^{\prime}, \alpha\right)} \times \\
\times\left\{\hat{i} \epsilon_{x}+\hat{j} \gamma\left[\epsilon_{y}-\frac{v(\vec{k} \times \hat{\epsilon})_{z}}{c k}\right]+\hat{k} \gamma\left[\epsilon_{z}+\frac{v(\vec{k} \times \hat{\epsilon})_{y}}{c k}\right]\right\}
\end{array}
$$

e

$$
\begin{array}{r}
\vec{B}^{\prime}\left(\vec{r}^{\prime}, t^{\prime}\right)=\Re e \sum_{\alpha=1}^{2} \int d^{3} k \mathfrak{h}(\vec{k}) e^{i \omega^{\prime} t^{\prime}-i \vec{k}^{\prime} \cdot \vec{r}^{\prime}-i \theta\left(\vec{k}^{\prime}, \alpha\right)} \times \\
\times\left\{\hat{i} \epsilon_{x}+\hat{j} \gamma\left[\frac{v \epsilon_{z}}{c}+\frac{(\vec{k} \times \hat{\epsilon})_{y}}{k}\right]+\hat{k} \gamma\left[\frac{(\vec{k} \times \hat{\epsilon})_{z}}{k}-\frac{v \epsilon_{y}}{c}\right]\right\}
\end{array}
$$

dado que $\hat{\epsilon}=\epsilon_{x} \hat{i}+\epsilon_{y} \hat{j}+\epsilon_{z} \hat{k}$. Devemos notar que as fases aleatórias são invariantes de Lorentz, $\log 0 \theta\left(\vec{k}^{\prime}, \alpha\right)=\theta(\vec{k}, \alpha)$. As componentes do vetor de onda e as freqüências angulares no referencial $S$ escritos nas expressões (2.7) e (2.8) devem ser substituídos pelas suas respectivas variáveis no referencial S', através das relações

$$
\begin{aligned}
& \omega^{\prime}=\gamma\left(\omega-v k_{x}\right), \\
& k_{x}^{\prime}=\gamma\left(k_{x}-\frac{v}{c^{2}} \omega\right), \\
& k_{y}^{\prime}=k_{y}, \\
& k_{z}^{\prime}=k_{z},
\end{aligned}
$$

sendo que

$$
\gamma=\frac{1}{\sqrt{1-\frac{v^{2}}{c^{2}}}}
$$

Assim como em (2.4), a densidade de energia também pode ser calculada no referencial S'. Para isso, a partir das equações (2.9), a relação entre os 
elementos de integração dos referenciais S e S' é

$$
d^{3} k^{\bullet}=d^{3} k^{\prime} \gamma\left(1+\frac{v k_{x}^{\prime}}{\omega^{\prime}}\right)
$$

Logo a densidade de energia no referencial S' é

$$
\begin{array}{r}
\frac{1}{8 \pi}\left\langle\vec{E}^{\prime 2}+\vec{B}^{\prime 2}\right\rangle=\frac{1}{8 \pi} \sum_{\alpha_{1}=1}^{2} \sum_{\alpha_{2}=1}^{2} \int d^{3} k_{1}^{\prime} \int d^{3} k_{2}^{\prime} \mathfrak{h}\left(\vec{k}_{1}\right) \mathfrak{h}\left(\vec{k}_{2}\right) \times \\
\times\left[\gamma^{2}\left(1+\frac{v k_{x 1}^{\prime}}{\omega_{1}^{\prime}}\right)^{2}\right]\left[\gamma^{2}\left(1+\frac{v k_{x 2}^{\prime}}{\omega_{2}^{\prime}}\right)^{2}\right] \delta_{\vec{k}_{1}^{\prime} \vec{k}_{2}^{\prime}} \delta_{\alpha_{1} \alpha_{2}}= \\
=\frac{1}{8 \pi} \sum_{\alpha=1}^{2} \int d^{3} k^{\prime} \gamma \mathfrak{h}^{2}(\omega)\left(1+\frac{v k_{x}^{\prime}}{\omega^{\prime}}\right) .
\end{array}
$$

A função $\mathfrak{h}$ depende de $\vec{k}$, sem linha, na expressão (2.12), pois estamos considerando que a distribuição espectral do campo de ponto-zero é invariante de Lorentz, então $\mathfrak{h}\left(\vec{k}^{\prime}\right)=\mathfrak{h}(\vec{k})$.

A hipótese de invariância relativística do espectro da radiação de pontozero implica que, no referencial $\mathrm{S}$, a densidade de energia contida num intervalo de freqüências $a \leq \omega \leq b$ deve ser igual à densidade de energia contida num intervalo de freqüências $a \leq \omega^{\prime} \leq b$, no referencial S'. Isto significa que, recorrendo às equações $(2.4)$ e (2.12),

$$
\int_{\omega=a}^{\omega=b} d^{3} k \mathfrak{h}^{2}(\omega)=\int_{\omega^{\prime}=a}^{\omega^{\prime}=b} d^{3} k^{\prime} \gamma \mathfrak{h}^{2}(\omega)\left(1+\frac{v k_{x}^{\prime}}{\omega^{\prime}}\right) .
$$

Como os valores $a$ e $b$ são arbitrários, podemos igualar os dois integrandos da equação (2.13). Levando em conta que as variáveis de integração são variáveis mudas, então a equação (2.13) implica que

$$
\mathfrak{h}^{2}\left(\omega^{\prime}\right)=\gamma \mathfrak{h}^{2}(\omega)\left(1+\frac{v k_{x}^{\prime}}{\omega^{\prime}}\right)
$$

Se a relação entre as freqüências angulares de cada referencial, dada em (2.9), é imposta à equação (2.14), então a função $\mathfrak{h}^{2}(\omega)$ deve depender linearmente de $\omega$. Para se determinar qual é o fator de proporcionalidade da função $\mathfrak{h}^{2}(\omega)$, é necessário recorrer a observação experimental. Há sistemas físicos que podem servir a essa verificação, por exemplo, o feito Casimir entre placas 
condutoras [12]. Logo podemos dizer que a energia de ponto-zero média de um modo normal de oscilação é $\frac{\hbar \omega}{2}$, tal que $\hbar$ é a constante de Planck divida por $2 \pi$. Em outras palavras, como a equação (2.6) está em coordenadas esféricas e foi integrada nos ângulos, então

$$
\pi^{2} \mathfrak{h}^{2}(\omega)=\frac{\hbar \omega}{2} .
$$

Pela definição (2.6), a distribuição espectral da radiação de ponto-zero é

$$
\rho(\omega)=\frac{\hbar \omega^{3}}{2 \pi^{2} c^{3}} .
$$

Devemos notar que as expressões (2.15) e (2.16) são as soluções com energia média $\frac{\hbar \omega}{2}$ por modo de oscilação para campos eletromagnéticos do vácuo, sendo imposto apenas a invariância de Lorentz.

\subsubsection{Radiação de Corpo Negro}

Outro resultado de importância fundamental obtido pela Eletrodinâmica Estocástica (SED) é a dedução da distribuição espectral da radiação de corpo negro. Através do método desenvolvido por Boyer [11], podemos obter o resultado bem conhecido da Mecânica Quântica para a radiação de corpo negro, porém sem assumir hipóteses quânticas. O cálculo da seção anterior, em que se obteve o espectro da radiação de ponto-zero $\rho(\omega)$, não levou em conta a temperatura do sistema. De fato devemos entender que, na expressão (2.16), foi obtido o espectro do campo eletromagnético do vácuo para uma temperatura nula, pois o espectro térmico da radiação de corpo negro não é invariante de Lorentz (veja as referências $[11,15]$ ). A radiação de corpo negro é um conjunto incoerente de modos de oscilação do campo eletromagnético excitados de acordo com uma temperatura $T$. Para a determinação do seu espectro, que vamos indicar por $\rho(\omega, T)$, consideram-se osciladores dipolares elétricos em interação com tal radiação.

Para resolver este problema, Einstein e Hopf [10] e posteriormente Boyer [11] estudaram as transferências de momento entre uma partícula e a radiação do meio. Vamos considerar uma partícula neutra polarizável com massa $M$ que tem uma parte oscilante de massa $m$ e carga $q(m \ll M)$ que se comporta como um oscilador elétrico dipolar. Em um dado instante $t$, a partícula possue um momento $p(t)=M v(t)$, sendo que $v(t)$ é a velocidade da 
partícula neste instante. Quando a temperatura é nula, a partícula interage apenas com a radiação de ponto-zero. Após um curto intervalo de tempo $\delta t$, um impulso aleatório $\Delta$ é transferido à partícula, devido às flutuações da radiação. Logo o momento da partícula no instante $t+\delta t$ é

$$
M v(t+\delta t)=M v(t)+\Delta, \quad \text { para } T=0 .
$$

Dado um ensemble de partículas sob as considerações acima, no equilíbrio, a média quadrática do momento no instante $t$ deve ser igual à mesma média quadrática do momento no instante $t+\delta t$, isto é,

$$
\left\langle(M v(t))^{2}\right\rangle_{T=0}=\left\langle(M v(t+\delta t))^{2}\right\rangle_{T=0}=\left\langle(M v(t)+\Delta)^{2}\right\rangle_{T=0} .
$$

Assim, necessariamente, segue que

$$
2 M\langle v \Delta\rangle_{T=0}=-\left\langle\Delta^{2}\right\rangle_{T=0} .
$$

Einstein e Hopf [10] consideraram que o termo $2 M\langle v \Delta\rangle_{T=0}$ fosse nulo, logo o termo $\left\langle\Delta^{2}\right\rangle_{T=0}$ também é nulo. Com isso o resultado obtido era a Lei de Rayleigh-Jeans. Para que a média quadrática do impulso da radiação de ponto-zero $\left\langle\Delta^{2}\right\rangle_{T=0}$ não fosse igual a zero, Boyer [11] considerou que uma partícula dentro de uma cavidade possue um termo de transferência de momento extra, que deve ser acrescentado na equação (2.17). Esse termo extra deve-se ao impacto mecânico da partícula contra as paredes da cavidade, transferindo o momento recebido do vácuo. Feito assim, a dedução da radiação de corpo negro se reduz a um caso espacialmente limitado. Aqui vamos seguir a proposta feita por de la Peña e Cetto [9], de que $2 M\langle v \Delta\rangle_{T=0}$ é diferente de zero. Seguindo os autores da referência [9], o impulso $\Delta$ em (2.17) é igual à variação do momento $M \delta v=M(v(t+\delta t)-v(t))$, logo a expressão (2.19) implica que o momento da partícula no instante $t$ é correlacionado com a sua variação no intervalo $(t, t+\delta t)$. Então a variável $M \delta v$ é um processo estocástico não markoviano, dado que o momento da partícula num certo instante depende dos instantes passados. Embora necessite estudos mais detalhados, este aspecto da interação da partícula com a radiação é esperado, visto que a reação da radiação, que age sobre o movimento da partícula, também depende das acelerações sofridas em tempos passados $[9,15,16]$.

Para uma temperatura diferente de zero, o momento da partícula após um curto intervalo de tempo $\delta t$ é

$$
M v(t+\tau)=M v(t)+\Delta-\eta v(t) \delta t
$$


sendo que $\eta v(t)$ é uma força de freamento da partícula, devido à radiação na qual a partícula está imersa. Esse efeito ocorre, porque, diferentemente da radiação de ponto-zero, que é postulada invariante de Lorentz, não há nenhuma condição de invariância relativística com relação à radiação térmica. De fato pode-se calcular que no referencial da partícula a radiação térmica é anisotrópica, provocando uma força contrária ao movimento da partícula (veja as referências $[11,15]$ ). Assim, para uma partícula com uma parte oscilante de massa $m$ pequena e carga $q$, então

$$
\eta=\frac{4 \pi^{2} q^{2}}{5 m c^{2}}\left(\rho(\omega, T)-\frac{\omega}{3} \frac{\partial \rho(\omega, T)}{\partial \omega}\right)
$$

sendo que $\omega$ é a freqüência de oscilação da parte oscilante da partícula neutra.

Prosseguindo como no caso da equação (2.17), no equilíbrio a média quadrática do momento $M v$ é

$$
\left\langle(M v(t))^{2}\right\rangle_{T}=\left\langle(M v(t+\tau))^{2}\right\rangle_{T}=\left\langle(M v(t)+\Delta-\eta v(t) \delta t)^{2}\right\rangle_{T}
$$

Esta equação leva a

$$
\left\langle\Delta^{2}\right\rangle_{T}+2 M\langle v \Delta\rangle_{T}-2 M \eta\left\langle v^{2}\right\rangle_{T} \delta t+\eta^{2}\left\langle v^{2}\right\rangle_{T}(\delta t)^{2}-2 \eta\langle v \Delta\rangle_{T} \delta t=0
$$

O comportamento não-markoviano da partícula devido ao termo $\langle v \Delta\rangle$ não é verificado para altas temperaturas, logo sua principal contribuição deve ter origem apenas na radiação de ponto-zero. Assim a aproximação

$$
\langle v \Delta\rangle_{T}=\langle v \Delta\rangle_{T=0}
$$

está de acordo para uma contribuição térmica desprezível.

A média quadrática do impulso fornecido à partícula, para $T \neq 0$, deve ser feita tomando a média sobre o ensemble das realizações do campo elétrico do vácuo, isto é, sobre suas fases aleatórias. Como o impulso é relevante apenas para a parte oscilante, pois a sua massa é pequena, então o resultado obtido é (veja as referências $[11,15])$

$$
\left\langle\Delta^{2}\right\rangle_{T}=\frac{8 \pi^{4} c q^{2}}{15 m \omega^{2}} \rho^{2}(\omega, T) \delta t .
$$

Devemos notar que o impulso depende em primeira ordem do intervalo $\delta t$.

Substituindo as expressões (2.19), (2.24) e (2.25) na equação (2.23), notamos que os dois últimos termos da equação $(2.23)$ dependem de $(\delta t)^{2}$. Logo, 
considerando intervalos de tempos muito pequenos, tais termos podem ser desprezados. Assim a equação que se obtem é

$$
\left\langle\Delta^{2}\right\rangle_{T}-\left\langle\Delta^{2}\right\rangle_{T=0}=2 M \eta\left\langle v^{2}\right\rangle_{T} \delta t .
$$

O segundo membro da equação acima deve satisfazer o princípio da equipartição,

$$
\frac{M}{2}\left\langle v^{2}\right\rangle_{T}=\frac{K T}{2}
$$

desde que se trate de uma partícula com uma massa total bastante grande. Neste caso o movimento da partícula tende a um movimento clássico, em que a contribuição da radiação de ponto-zero é insignificante. Nesse limite o segundo termo do primeiro membro da equação (2.26) tende a zero, garantindo o uso de (2.27).

Com as expressões (2.21), (2.25) e (2.27), a equação (2.26) torna-se uma equação diferencial:

$$
\frac{\pi^{2} c^{3}}{3 K T \omega^{2}}\left[\rho^{2}(\omega, T)-\left(\frac{\hbar \omega^{3}}{2 \pi^{2} c^{3}}\right)^{2}\right]=\rho(\omega, T)-\frac{\omega}{3} \frac{\partial \rho(\omega, T)}{\partial \omega} .
$$

A solução desta equação diferencial é

$$
\rho(\omega, T)=\frac{\omega^{2}}{\pi^{2} c^{3}}\left(\frac{\hbar \omega}{e^{\frac{\hbar \omega}{K T}}-1}+\frac{\hbar \omega}{2}\right)=\frac{\hbar \omega^{3}}{2 \pi^{2} c^{3}} \operatorname{coth}\left(\frac{\hbar \omega}{2 K T}\right),
$$

que é a distribuição de Planck completa, inclusos os termos térmicos e de ponto-zero. Esta distribuição espectral da radiação de corpo negro é também obtida pela Eletrodinâmica Quântica.

\subsection{Funções de Correlação para Campos Ele- tromagnéticos Livres}

Demonstrado o sucesso da SED no cálculo do espectro da radiação de corpo negro, uma conseqüência natural seria analisar as propriedades estatísticas dos campos eletromagnéticos livres no vácuo. Os cálculos dos momentos estatísticos (por exemplo, a média e a média quadrática) das variáveis dinâmicas de um sistema nos dariam informações suficientes para comparar a SED com outras teorias e fornecer um meio para sua verificação experimental. Então, 
para uma abordagem geral, devemos analizar as funções de correlação dos campos elétricos e magnéticos flutuantes no vácuo. Nesta seção, são apresentados os cálculos necessários para se obter essas funções de correlação, usando-se a SED e a Eletrodinâmica Quântica (QED) [14]. Os resultados obtidos a seguir estabelecem uma relação clara entre a QED e a SED, além de serem importantes para o estudo das interações do circuito RLC com o meio e com um dipolo elétrico, apresentado nos próximos capítulos.

Inicialmente vamos calcular as funções de correlação pela SED, como foram feitas por Boyer [14]. Os campos eletromagnéticos livres (térmicos e de ponto-zero) satisfazem as equações de Maxwell homogêneas e vamos utilizar o gauge de Coulomb. Assim podemos expandir o campo elétrico em uma soma de ondas planas como em (2.1), similarmente, o campo magnético pode ser expresso por (2.2), notando que as amplitudes das expansões devem depender agora também da temperatura $T$, isto é, $\mathfrak{h}=\mathfrak{h}(\vec{k}, T)$.

Para facilitar os cálculos, podem-se definir as variáveis

$$
\begin{array}{r}
a(\vec{k}, \alpha) \equiv e^{i \theta(\vec{k}, \alpha)}, \\
a^{*}(\vec{k}, \alpha) \equiv e^{-i \theta(\vec{k}, \alpha)},
\end{array}
$$

de modo que substituindo nas expressões (2.1) e (2.2), o campo elétrico fica

$$
\vec{E}(\vec{r}, t)=\sum_{\alpha=1}^{2} \int d^{3} k \hat{\epsilon}(\vec{k}, \alpha) \frac{\mathfrak{h}(\vec{k}, T)}{2}\left[a(\vec{k}, \alpha) e^{-i \omega t+i \vec{k} \cdot \vec{r}}+a^{*}(\vec{k}, \alpha) e^{i \omega t-i \vec{k} \cdot \vec{r}}\right]
$$

e também o campo magnético fica

$$
\vec{B}(\vec{r}, t)=\sum_{\alpha=1}^{2} \int d^{3} k \frac{\vec{k} \times \hat{\epsilon}(\vec{k}, \alpha)}{k} \frac{\mathfrak{h}(\vec{k}, T)}{2}\left[a(\vec{k}, \alpha) e^{-i \omega t+i \vec{k} \cdot \vec{r}}+a^{*}(\vec{k}, \alpha) e^{i \omega t-i \vec{k} \cdot \vec{r}}\right] .
$$

Esta forma de escrever os campos elétrico (2.31) e magnético (2.32) tem a vantagem de que podemos compará-los com suas respectivas expressões quânticas (2.39) e (2.40).

A amplitude $\mathfrak{h}$ dos campos elétricos e magnéticos pode ser determinada, notando que $\pi^{2} \mathfrak{h}^{2}(\vec{k}, \alpha, T)$ é, dado um banho térmico à temperatura $T$, a energia por modo normal de oscilação, a qual pode ser relacionada com a expressão do espectro de corpo negro (2.29) através da definição (2.6). Logo

$$
\pi^{2} \mathfrak{h}^{2}(\vec{k}, T)=\frac{\hbar \omega}{2} \operatorname{coth}\left(\frac{\hbar \omega}{2 K T}\right)
$$


Conhecidas as expressões dos campos, é possivel calcular suas funções de correlação efetuando as médias sobre as fases aleatórias $\theta(\vec{k}, \alpha)$. Assim, são de particular interesse as seguintes funções de correlação entre as variáveis $a(\vec{k}, \alpha)$ e $a^{*}(\vec{k}, \alpha)$

$$
\begin{gathered}
\left\langle a(\vec{k}, \alpha) a\left(\vec{k}^{\prime}, \alpha^{\prime}\right)\right\rangle=\left\langle a^{*}(\vec{k}, \alpha) a^{*}\left(\vec{k}^{\prime}, \alpha^{\prime}\right)\right\rangle=0, \\
\left\langle a(\vec{k}, \alpha) a^{*}\left(\vec{k}^{\prime}, \alpha^{\prime}\right)\right\rangle=\left\langle a^{*}(\vec{k}, \alpha) a\left(\vec{k}^{\prime}, \alpha^{\prime}\right)\right\rangle=\delta_{\alpha \alpha^{\prime}} \delta_{\vec{k} \vec{k}^{\prime}} .
\end{gathered}
$$

Aplicando estes resultados nas expressões (2.31) e (2.32), podemos calcular as funções de correlação dos campos elétrico e magnético. A função de correlação para as componentes $i$ e $j$ quaisquer do campo elétrico $\vec{E}$ é:

$$
\begin{aligned}
\left\langle E_{i}\left(\vec{r}_{1}, t_{1}\right) E_{j}\left(\vec{r}_{2}, t_{2}\right)\right\rangle_{T} & =\int d^{3} k\left(\delta_{i j}-\frac{k_{i} k_{j}}{k^{2}}\right) \frac{\hbar \omega}{8 \pi^{2}} \operatorname{coth}\left(\frac{\hbar \omega}{2 K T}\right) \times \\
& \times\left[e^{-i \omega\left(t_{1}-t_{2}\right)+i \vec{k} \cdot\left(\vec{r}_{1}-\vec{r}_{2}\right)}+e^{i \omega\left(t_{1}-t_{2}\right)-i \vec{k} \cdot\left(\vec{r}_{1}-\vec{r}_{2}\right)}\right] .
\end{aligned}
$$

A função de correlação do campo magnético é igual ao caso do campo elétrico:

$$
\left\langle E_{i}\left(\vec{r}_{1}, t_{1}\right) E_{j}\left(\vec{r}_{2}, t_{2}\right)\right\rangle_{T}=\left\langle B_{i}\left(\vec{r}_{1}, t_{1}\right) B_{j}\left(\vec{r}_{2}, t_{2}\right)\right\rangle_{T} .
$$

A função de correlação entre o campo elétrico e magnético é:

$$
\begin{array}{r}
\left\langle E_{i}\left(\vec{r}_{1}, t_{1}\right) B_{j}\left(\vec{r}_{2}, t_{2}\right)\right\rangle_{T}=\int d^{3} k \epsilon_{i j l} \frac{k_{l}}{k} \frac{\hbar \omega}{8 \pi^{2}} \operatorname{coth}\left(\frac{\hbar \omega}{2 K T}\right) \times \\
\times\left[e^{-i \omega\left(t_{1}-t_{2}\right)+i \vec{k} \cdot\left(\vec{r}_{1}-\vec{r}_{2}\right)}+e^{i \omega\left(t_{1}-t_{2}\right)-i \vec{k} \cdot\left(\vec{r}_{1}-\vec{r}_{2}\right)}\right],
\end{array}
$$

sendo que

$$
\epsilon_{i j l}=\left\{\begin{array}{cl}
1 & , \text { se }(i j l) \text { for permutação par de }(x y z) \\
-1 & , \text { se }(i j l) \text { for permutação impar de }(x y z) \\
0 & , \text { se }(i j l) \text { tiver dois índices iguais }
\end{array}\right.
$$

Passamos agora a calcular as funções de correlação dos campos pela QED, seguindo Boyer [14]. Para clareza, exceto quando mencionado, todos os operadores quânticos são indicados por letras grifadas. Novamente os campos seguem as equações de Maxwell e o gauge de Coulomb. Os campos elétrico e magnético podem ser quantizados da forma convencional [15, 17], pela qual o operador de campo elétrico $\underline{\underline{E}}$ é

$$
\underline{\vec{E}}(\vec{r}, t)=\sum_{\alpha=1}^{2} \int d^{3} k \hat{\epsilon}(\vec{k}, \alpha) \frac{\sqrt{\hbar \omega}}{2 \pi}\left[\underline{a}(\vec{k}, \alpha) e^{-i \omega t+i \vec{k} \cdot \vec{r}}+\underline{a}^{\dagger}(\vec{k}, \alpha) e^{i \omega t-i \vec{k} \cdot \vec{r}}\right],
$$


e similarmente o operador de campo magnético $\underline{B}$ é

$$
\underline{\vec{B}}(\vec{r}, t)=\sum_{\alpha=1}^{2} \int d^{3} k \frac{\vec{k} \times \hat{\epsilon}(\vec{k}, \alpha)}{k} \frac{\sqrt{\hbar \omega}}{2 \pi}\left[\underline{a}(\vec{k}, \alpha) e^{-i \omega t+i \vec{k} \cdot \vec{r}}+\underline{a}^{\dagger}(\vec{k}, \alpha) e^{i \omega t-i \vec{k} \cdot \vec{r}}\right],
$$

sendo que as variáveis $\vec{k}, \hat{\epsilon}(\vec{k}, \alpha)$ e $\alpha$ já foram definidas anteriormente.

Com as expressões (2.39) e (2.40), a hamiltoniana para os campos eletromagnéticos livres fica

$$
H_{F V}=\frac{1}{8 \pi} \int d^{3} r\left(\underline{\vec{E}}^{2}(\vec{r}, t)+\underline{\vec{B}}^{2}(\vec{r}, t)\right)=\sum_{\alpha=1}^{2} \int d^{3} k \hbar \omega\left(\underline{a}^{\dagger}(\vec{k}, \alpha) \underline{a}(\vec{k}, \alpha)+\frac{1}{2}\right),
$$

da qual $\underline{a}$ e $\underline{a}^{\dagger}$ são os operadores de aniquilação e criação dos autoestados completos e ortonormais $\left|n_{\vec{k} \alpha}\right\rangle$ sobre um espaço de Hilbert. Estes operadores seguem as seguintes relações de comutação:

$$
\begin{gathered}
{\left[\underline{a}(\vec{k}, \alpha), \underline{a}\left(\overrightarrow{k^{\prime}}, \alpha^{\prime}\right)\right]=\left[\underline{a}^{\dagger}(\vec{k}, \alpha), \underline{a}^{\dagger}\left(\overrightarrow{k^{\prime}}, \alpha^{\prime}\right)\right]=0} \\
{\left[\underline{a}(\vec{k}, \alpha), \underline{a}^{\dagger}\left(\overrightarrow{k^{\prime}}, \alpha^{\prime}\right)\right]=\delta_{\alpha \alpha^{\prime}} \delta_{\vec{k} \vec{k}^{\prime}}}
\end{gathered}
$$

Desse modo valem as equações

$$
\begin{gathered}
\underline{a}(\vec{k}, \alpha)\left|n_{\vec{k} \alpha}\right\rangle=\sqrt{n_{\vec{k} \alpha}}\left|n_{\vec{k} \alpha}-1\right\rangle, \\
\underline{a}^{\dagger}(\vec{k}, \alpha)\left|n_{\vec{k} \alpha}\right\rangle=\sqrt{n_{\vec{k} \alpha}+1}\left|n_{\vec{k} \alpha}+1\right\rangle
\end{gathered}
$$

e $\underline{a}(\vec{k}, \alpha)|0\rangle=0$, sendo que $n_{\vec{k} \alpha} \in\{0,1,2,3, \ldots\}$. O autovalor de energia $W_{n}(\vec{k}, \alpha)$ do autoestado $\left|n_{\vec{k} \alpha}\right\rangle$ é

$$
W_{n}(\vec{k}, \alpha)=\hbar \omega\left(n_{\vec{k} \alpha}+\frac{1}{2}\right)
$$

Considerando que o banho de radiação térmica está a uma temperatura $T$, então o peso estatístico para uma certa autoenergia $W_{n}(\vec{k}, \alpha)$ é

$$
e^{-\frac{W_{n}(\vec{k}, \alpha)}{K T}}
$$

Assim pode-se escrever que a função de partição do sistema é

$$
\Xi=\sum_{n_{\vec{k}, \alpha}=0}^{\infty} e^{-\frac{\hbar \omega\left(n_{\bar{k} \alpha}+\frac{1}{2}\right)}{K T}}
$$


Para se obter a função de correlação entre duas variáveis de campos no caso quântico, deve-se inicialmente calcular os valores esperados do produto dos respectivos operadores para cada autoestado de cada operador. Assim a função de correlação é a média desses valores esperados, ponderada pelas funções de partição de cada variável de campo. Para o caso das componentes $i$ e $j$ quaisquer do campo elétrico $\underline{\vec{E}}$, temos

$$
\begin{array}{r}
\left\langle\left|\underline{E_{i}}\left(\vec{r}_{1}, t_{1}\right) \underline{E_{j}}\left(\vec{r}_{2}, t_{2}\right)\right|\right\rangle_{T}=\sum_{n_{\overrightarrow{k_{1} \alpha_{1}}}=0}^{\infty} \sum_{n_{\overrightarrow{k_{2}} \alpha_{2}}=0}^{\infty} \frac{e^{-\frac{w_{n}\left(\overrightarrow{k_{1}, \alpha_{1}}\right)}{K T}}}{\Xi_{1}} \frac{e^{-\frac{w_{n}\left(\overrightarrow{k_{2}, \alpha_{2}}\right)}{K T}}}{\Xi_{2}} \times \\
\times\left\langle n_{\overrightarrow{k_{1} \alpha_{1}}} n_{\overrightarrow{k_{2}} \alpha_{2}}\left|\underline{E_{i}}\left(\overrightarrow{r_{1}}, t_{1}\right) \underline{E_{j}}\left(\overrightarrow{r_{2}}, t_{2}\right)\right| n_{\overrightarrow{k_{1} \alpha_{1}}} n_{\overrightarrow{k_{2}} \alpha_{2}}\right\rangle,
\end{array}
$$

sendo que os termos $\Xi_{1}$ e $\Xi_{2}$ são as respectivas funções de partição de cada variável de campo da função de correlação. $\mathrm{O}$ autoestado $\left|n_{\overrightarrow{k_{1}} \alpha_{1}} n_{\overrightarrow{k_{2}} \alpha_{2}}\right\rangle$ é o produto direto do autoestado $\left|n_{\overrightarrow{k_{1} \alpha_{1}}}\right\rangle$ que contem os $n_{\overrightarrow{k_{1} \alpha_{1}}}$ fótons do campo $\underline{E_{i}}\left(\vec{r}_{1}, t_{1}\right)$ com o autoestado $\left|n_{\overrightarrow{k_{2} \alpha_{2}}}\right\rangle$ que contem $n_{\overrightarrow{k_{2} \alpha_{2}}}$ fótons do campo $\underline{E_{j}}\left(\vec{r}_{2}, t_{2}\right)$. O valor esperado dos operadores de campos é

$$
\begin{array}{r}
\left\langle n_{\overrightarrow{k_{1} \alpha_{1}}} n_{\overrightarrow{k_{2} \alpha_{2}}}\left|\underline{E_{i}}\left(\overrightarrow{r_{1}}, t_{1}\right) \underline{E_{j}}\left(\overrightarrow{r_{2}}, t_{2}\right)\right| n_{\overrightarrow{k_{1} \alpha_{1}}} n_{\overrightarrow{k_{2} \alpha_{2}}}\right\rangle=\sum_{\alpha=1}^{2} \int d^{3} k \epsilon_{i} \epsilon_{j} \frac{\hbar}{4 \pi^{2}} \times \\
\times\left[\left(n_{\vec{k} \alpha}\right) e^{-i \omega\left(t_{1}-t_{2}\right)+i \vec{k} \cdot\left(\overrightarrow{r_{1}}-\overrightarrow{r_{2}}\right)}+\left(n_{\vec{k} \alpha}+1\right) e^{i \omega\left(t_{1}-t_{2}\right)-i \vec{k} \cdot\left(\overrightarrow{r_{1}}-\overrightarrow{r_{2}}\right)}\right] .
\end{array}
$$

Substituindo a equação (2.48) em (2.47), e trocando a ordem da soma com a integração, temos

$$
\begin{array}{r}
\left\langle\underline{E_{i}}\left(\vec{r}_{1}, t_{1}\right) \underline{E_{j}}\left(\vec{r}_{2}, t_{2}\right) \mid\right\rangle_{T}=\int d^{3} k\left(\delta_{i j}-\frac{k_{i} k_{j}}{k^{2}}\right) \frac{1}{4 \pi^{2} \Xi} \sum_{n=0}^{\infty} \hbar \omega e^{-\frac{\hbar \omega\left(n+\frac{1}{2}\right)}{K T}} \times \\
\times\left[\left(n_{\vec{k} \alpha}\right) e^{-i \omega\left(t_{1}-t_{2}\right)+i \vec{k} \cdot\left(\vec{r}_{1}-\vec{r}_{2}\right)}+\left(n_{\vec{k} \alpha}+1\right) e^{i \omega\left(t_{1}-t_{2}\right)-i \vec{k} \cdot\left(\vec{r}_{1}-\vec{r}_{2}\right)}\right] .
\end{array}
$$

Fazendo o mesmo para o campo magnético, temos

$$
\left\langle\underline{E_{i}}\left(\vec{r}_{1}, t_{1}\right) \underline{E_{j}}\left(\vec{r}_{2}, t_{2}\right) \mid\right\rangle_{T}=\left\langle\left|\underline{B_{i}}\left(\vec{r}_{1}, t_{1}\right) \underline{B_{j}}\left(\vec{r}_{2}, t_{2}\right)\right|\right\rangle_{T} .
$$

$E$ a função de correlação entre o campo elétrico e o campo magnético é

$$
\begin{array}{r}
\left\langle\underline{\underline{E_{i}}}\left(\vec{r}_{1}, t_{1}\right) \underline{B_{j}}\left(\vec{r}_{2}, t_{2}\right) \mid\right\rangle_{T}=\int d^{3} k \epsilon_{i j l} \frac{k_{l}}{k} \frac{1}{4 \pi^{2} \Xi} \sum_{n=0}^{\infty} \hbar \omega e^{-\frac{\hbar \omega\left(n+\frac{1}{2}\right)}{K T}} \times \\
\quad \times\left[\left(n_{\vec{k} \alpha}\right) e^{-i \omega\left(t_{1}-t_{2}\right)+i \vec{k} \cdot\left(\vec{r}_{1}-\vec{r}_{2}\right)}+\left(n_{\vec{k} \alpha}+1\right) e^{i \omega\left(t_{1}-t_{2}\right)-i \vec{k} \cdot\left(\vec{r}_{1}-\vec{r}_{2}\right)}\right] .
\end{array}
$$


Podemos notar que as expressões (2.35), (2.36) e (2.37) não são iguais às expressões (2.49), (2.50) e (2.51), respectivamente. Os termos de radiação térmica e de ponto-zero são notados claramente nas funções de correlação calculadas pela SED, mas não o são nas funções de correlação calculadas pela QED. Isto deve-se a importância da ordem dos operadores nas teorias quânticas, que em geral não comutam entre si. Por outro lado as teorias quânticas afirmam que todo operador hermitiano de um sistema físico corresponde a um observável físico. Para resolver este problema devemos simetrizar os operadores quânticos pelo ordenamento de Weyl [24], que estabelece uma correspondência entre os operadores quânticos e as variáveis ordinárias de observáveis físicos. No caso das funções de correlação dos campos elétricos (2.35) e (2.49), a correspondência entre a SED e a QED é verificada por

$$
\left\langle E_{i}\left(\vec{r}_{1}, t_{1}\right) E_{j}\left(\vec{r}_{2}, t_{2}\right)\right\rangle_{T}=\frac{1}{2}\left\langle\left|\underline{E_{i}}\left(\vec{r}_{1}, t_{1}\right) \underline{E_{j}}\left(\vec{r}_{2}, t_{2}\right)+\underline{E_{j}}\left(\vec{r}_{2}, t_{2}\right) \underline{E_{i}}\left(\vec{r}_{1}, t_{1}\right)\right|\right\rangle_{T} .
$$

A relação entre as funções de correlação dos campos magnéticos do caso clássico e do caso quântico (respectivamente (2.36) e (2.50)) são análogas à equação (2.52). A mesma analogia se faz para as funções de correlação mistas de campos elétricos e magnéticos (veja as expressões (2.37) e (2.51)). Dentro do âmbito desta dissertação, apresentamos apenas as funções de correlação de duas variáveis de campo, pois vamos restringir-nos aos processos estocásticos com ruído gaussiano, de modo que a média e a média quadrática da variável estocástica determinam completamente suas densidades de probabilidades [18]. Entretanto é possível mostrar que, no caso geral, as funções de correlação da SED com um número arbitrário de variáveis de campo são iguais às respectivas funções de correlação da QED com seus operadores de campo simetrizados. A demostração dessa asserção é encontrada na referência [14]. Isso estabelece uma equivalência estatística completa entre a SED e a QED para campos eletromagnéticos livres, visto que os valores esperados dos observáveis físicos podem ser calculados através dessas funções de correlação.

\subsection{Oscilador Elétrico Dipolar}

Assim como no caso dos campos eletromagnéticos livres, é possível encontrar uma equivalência entre a SED e a QED no caso de uma carga elétrica sujeita a ação de um potencial harmônico e dos campos eletromagnéticos livres. A exemplo da seção anterior, apresentamos aqui os métodos da SED e da QED 
para calcular os momentos estatísticos das variáveis dinâmicas do oscilador elétrico dipolar (posição e seu momento canônico).

Conforme as referências $[14,15,16]$, vamos considerar uma carga $q$ com massa $m_{0}$, que oscila em torno da posição $\vec{r}_{0}$, sofrendo a ação de um potencial harmônico com freqüência natural $\omega_{0}$. A carga oscilante também está sob a ação dos campos elétrico $\vec{E}_{R}(\vec{r}, t)$ e magnético $\vec{B}_{R}(\vec{r}, t)$. A hamiltoniana do sistema completo, usando o acoplamento mínimo, é

$$
H=\frac{1}{2 m_{0}}\left(\vec{p}-\frac{q}{c} \vec{A}_{R}(\vec{r}, t)\right)^{2}+\frac{1}{2} m_{0} \omega_{0}^{2} \vec{x}^{2}+\frac{1}{8 \pi} \int d^{3} r\left(\vec{E}_{R}^{2}(\vec{r}, t)+\vec{B}_{R}^{2}(\vec{r}, t)\right)
$$

para a qual foi definido o potencial vetor de forma que

$$
\vec{E}_{R}=-\vec{\nabla} \Phi-\frac{1}{c} \frac{\partial \vec{A}_{R}}{\partial t}
$$

dado o potencial escalar $\Phi$, que não é considerado uma variável dinâmica, e

$$
\vec{B}_{R}=\vec{\nabla} \times \vec{A}_{R}
$$

As variáveis $\vec{x}$ e $\vec{p}$ são a posição e o momento canônico da carga oscilante, respectivamente.

A hamiltoniana (2.53) pode ser usada tanto no formalismo clássico quanto no formalismo quantum-mecânico, tomada as devidas mudanças de variáveis clássicas para operadores quânticos. A partir da hamiltoniana (2.53), podemos aplicar as equações clássicas de Hamilton, $\dot{x}_{i}=\frac{\partial H}{\partial p_{i}}$ e $\dot{p}_{i}=-\frac{\partial H}{\partial x_{i}}$, no caso clássico, ou aplicar as equações de Heisenberg, $\dot{x}_{i}=\frac{1}{i \hbar}\left[\underline{x_{i}}, \underline{H}\right]$ e $\underline{\dot{p}_{i}}=\frac{1}{i \hbar}\left[\underline{p_{i}}, \underline{H}\right]$, no caso quântico. As equações obtidas em ambos formalismos são formalmente idênticas. Para o caso clássico, obtêm-se

$$
\dot{\vec{x}}=\frac{1}{m_{0}}\left(\vec{p}-\frac{q}{c} \vec{A}_{R}\right)
$$

e

$$
\begin{aligned}
\dot{\vec{p}} & =-\frac{1}{2 m_{0}} \vec{\nabla}\left(\vec{p}-\frac{q}{c} \vec{A}_{R}\right)^{2}-m_{0} \omega_{0}^{2} \vec{x}= \\
& =\frac{q}{c}(\dot{\vec{x}} \cdot \vec{\nabla}) \vec{A}_{R}+\frac{q}{c} \dot{\vec{x}} \times \vec{B}_{R}-m_{0} \omega_{0}^{2} \vec{x}
\end{aligned}
$$

No caso quântico, as equações são as mesmas, no entanto deve-se ler as variáveis $\vec{x}, \vec{p}, \vec{A}_{R}$ e $\vec{B}_{R}$ como operadores. 
Derivando-se com relação ao tempo a equação (2.56) e substituindo pela equação (2.57), obtem-se que

$$
\begin{aligned}
m_{0} \ddot{\vec{x}} & =\left(\dot{\vec{p}}-\frac{q}{c} \dot{\vec{A}}\right)= \\
& =q \vec{E}_{R}+\frac{q}{c} \dot{\vec{x}} \times \vec{B}_{R}-m_{0} \omega_{0}^{2} \vec{x} .
\end{aligned}
$$

Consistente com um movimento não relativístico, a amplitude espacial das oscilações do dipolo elétrico é muito menor que os comprimentos de onda relevantes dos campos. Logo faz-se a aproximação de que os campos eletromagnéticos atuam apenas na posição de equilíbrio do oscilador $\vec{r}_{0}$, como conseqüência a contribuição do termo do campo magnético em (2.58) é desprezível. Assim a equação (2.58) torna-se

$$
m_{0} \ddot{\vec{x}}+m_{0} \omega_{0}^{2} \vec{x} \simeq q \vec{E}_{R}\left(\vec{r}_{0}, t\right) .
$$

É possível mostrar que o campo elétrico $\vec{E}_{R}\left(\vec{r}_{0}, t\right)$ possue duas contribuições (veja as referências $[9,15]$ ), uma parte é devido ao campo elétrico livre $\vec{E}\left(\vec{r}_{0}, t\right)$, que pode ser expresso como em $(2.31)$, no caso da SED, ou pode ser expresso como em (2.39), no caso da QED. A outra contribuição é devida ao campo elétrico da reação da zadiação $\vec{E}_{R R}(t)$. A equação (2.59) pode ser melhor escrita como

$$
m_{0} \ddot{\vec{x}}+m_{0} \omega_{0}^{2} \vec{x}=\vec{E}\left(r_{0}, t\right)+\vec{E}_{R R}(t),
$$

válida tanto classicamente como quanticamente.

O campo da reação da radiação pode ser calculado pelo formalismo clássico $[9,16]$ ou pelo formalismo quântico [15]. Em ambos formalismos as expressões do campo da reação da radiação são formalmente iguais, lembrando que $\vec{E}_{R R}$ deve ser uma variável clássica pela SED ou um operador quântico pela QED. Logo

$$
\vec{E}_{R R}(t)=\frac{2 q}{3 c^{3}} \dddot{\vec{x}}-\frac{\delta m}{q} \ddot{\vec{x}}(t)
$$

sendo que $\delta m$ é a contribuição de massa eletromagnética, devido à energia do campo eletromagnético da carga. Assim a massa observada da carga $m$ deve ser renormalizada devido à contribuição de $\delta m$. Rearranjando os termos em (2.60), com o uso de (2.61), pode-se escrever que $m=m_{0}+\delta m$, sendo que $m$ é a massa observada experimentalmente. 
A equação (2.60) é a equação do movimento do oscilador dipolar elétrico tridimensional, formalmente válida para a SED e para a QED. Por simplificação, sem perder a generalidade do sistema físico, vamos estudar o movimento de um oscilador unidimensional com posição $x$. O caso unidimensional da equação (2.60) é

$$
m \ddot{x}+m \omega_{0}^{2} x-\frac{2 q^{2}}{3 c^{3}} \dddot{x}=q E_{x}\left(\vec{r}_{0}, t\right),
$$

sendo que $E_{x}\left(\vec{r}_{0}, t\right)$ é a componente do campo elétrico flutuante na direção do movimento do oscilador (veja a expressão (2.31) no caso clássico e a expressão (2.39) no caso quântico). A partir deste ponto, vamos novamente distingüir os operadores quânticos das variáveis clássicas grifando-os.

A solução estacionária da equação (2.62) é obtida pela sua transformada de Fourier. Pela SED, utilizando a expressão (2.31), a posição do oscilador é

$$
x=\frac{q}{m} \sum_{\alpha=1}^{2} \int d^{3} k \frac{(\vec{k}, \alpha, T)}{2}\left[\frac{a(\vec{k}, \alpha)}{C} e^{-i \omega t+i \vec{k} \cdot \vec{r}_{0}}+\frac{a^{*}(\vec{k}, \alpha)}{C^{*}} e^{i \omega t-i \vec{k} \cdot \vec{r}_{0}}\right] .
$$

E pela QED, utilizando a expressão (2.39), o operador posição é

$$
\underline{x}=\frac{q}{m} \sum_{\alpha=1}^{2} \int d^{3} k \frac{\sqrt{\hbar \omega}}{2 \pi}\left[\frac{a(\vec{k}, \alpha)}{C} e^{-i \omega t+i \vec{k} \cdot \overrightarrow{r_{0}}}+\frac{\underline{a}^{\dagger}(\vec{k}, \alpha)}{C^{*}} e^{i \omega t-i \ddot{k} \overrightarrow{r_{0}}}\right] .
$$

Para as equações (2.63) e (2.64), temos que

$$
\begin{aligned}
& C(\omega)=-\omega^{2}+\omega_{0}^{2}-i \Gamma \omega^{3}, \\
& \Gamma=\frac{2 q^{2}}{3 m c^{3}} .
\end{aligned}
$$

Obtido as soluções $x$, pela SED, e $\underline{x}$, pela QED, os respectivos momentos canônicos $p$ e $\underline{p}$ são determinados de forma similar ao caso tridimensional, dado pela equação (2.56). No caso unidimensional, o momento pela SED é

$$
p=m \dot{x}+\frac{q}{c} A_{x}(t)
$$

O momento segundo a QED, similar à equação (2.66), é dado por $\underline{p}=m \underline{\dot{x}}+$ ${ }_{c}^{q} A_{x}(t)$.

Determinados as posições e os momentos, pode-se calcular os valores médios para qualquer potência destas variáveis, além de podermos calcular o valor médio de composições da posição com o momento, no âmbito de 
cada formalismo [14]. Os valores médios da primeira potência da posição ou do momento são nulos, tanto na SED quanto na QED, ou seja, $\langle x\rangle_{T}=$ $\langle\underline{x}\rangle_{T}=\langle p\rangle_{T}=\langle\underline{p}\rangle_{T}=0$. No entanto para outras ordens de potências os valores médios não são nulos. Por exemplo, usando a SED, a média quadrática da posição é

$$
\begin{aligned}
\left\langle x^{2}\right\rangle_{T}=\frac{q^{2}}{m^{2}} \sum_{\alpha_{1}=1}^{2} \sum_{\alpha_{2}=1}^{2} & \int d^{3} k_{1} \int d^{3} k_{2} \epsilon_{x_{1}} \epsilon_{x_{2}} \frac{\mathfrak{h}\left(\vec{k}_{1}, \alpha_{1}, T\right)}{2} \frac{\mathfrak{h}\left(\vec{k}_{2}, \alpha_{2}, T\right)}{2} \times \\
& \times\left\langle\left(\frac{a_{1}}{C\left(\omega_{1}\right)} e^{-i \omega_{1} t+i \vec{k}_{1} \cdot \vec{r}_{0}}+\frac{a_{1}^{*}}{C^{*}\left(\omega_{1}\right)} e^{i \omega_{1} t-i \vec{k}_{1} \cdot \vec{r}_{0}}\right) \times\right. \\
& \left.\times\left(\frac{a_{2}}{C\left(\omega_{2}\right)} e^{-i \omega_{2} t+i \vec{k}_{2} \cdot \vec{r}_{0}}+\frac{a_{2}^{*}}{C^{*}\left(\omega_{2}\right)} e^{i \omega_{2} t-i \vec{k}_{2} \cdot \vec{r}_{0}}\right)\right\rangle
\end{aligned}
$$

sendo que as médias são feitas sobre as fases aleatórias. Tomando as relações (2.34) e integrando nos ângulos, obtemos

$$
\left\langle x^{2}\right\rangle_{T}=\frac{4 \pi q^{2}}{3 m^{2} c^{3}} \int_{0}^{\infty} d \omega \frac{\omega^{2} \mathfrak{h}^{2}(\omega, T)}{\left(\omega^{2}-\omega_{0}^{2}\right)^{2}+\Gamma^{2} \omega^{6}} .
$$

No limite $\Gamma \omega_{0}=\frac{2 q^{2} \omega_{0}}{3 m c^{3}} \ll 1$, nota-se que o integrando da expressão (2.68) tem um pico muito acentuado para valores de $\omega$ próximos de $\omega_{0}$. Assim, a maior contribuição da integral em (2.68) vem da região em torno desse pico, de modo que podemos considerar $\omega^{2} \mathfrak{h}^{2}(\omega, T) \simeq \omega_{0}^{2} \mathfrak{h}^{2}\left(\omega_{0}, T\right)$. Logo a expressão (2.68) torna-se (veja as referências $[14,15])$

$$
\left\langle x^{2}\right\rangle_{T} \simeq \frac{4 \pi q^{2}}{3 m^{2} c^{3}} \mathfrak{h}^{2}\left(\omega_{0}, T\right) \int_{0}^{\infty} \frac{d \omega}{4\left(\omega-\omega_{0}\right)^{2}+\Gamma^{2} \omega_{0}^{4}}=\frac{\hbar}{2 m \omega_{0}} \operatorname{coth}\left(\frac{\hbar \omega_{0}}{2 K T}\right) .
$$

Essa aproximação é muito boa para o caso de osciladores dipolares elétricos de escala atômica ou molecular, em que vale $\Gamma \omega_{0} \ll 1$. Esse limite também serve para descrever o caso de um oscilador harmônico neutro, no entanto os efeitos da radiação térmica e de ponto-zero permanecem e determinam o comportamento do oscilador. Esse é um resultado relevante da SED, visto que os valores obtidos por esta teoria são claramente diferentes do caso da Mecânica Clássica. Dentro do limite $\Gamma \omega_{0} \ll 1$, os valores médios da posição 
$x$ do oscilador dipolar para as várias ordens de potência são, pela SED, [14]

$$
\begin{aligned}
& \left\langle x^{2 j}\right\rangle_{T}=\frac{(2 j) !}{j ! 2^{j}}\left(\frac{\hbar}{2 m \omega_{0}}\right)^{j} \sum_{n_{1}=0}^{\infty} \sum_{n_{2}=0}^{\infty} \cdots \sum_{n_{j}=0}^{\infty}\left(n_{1}+\frac{1}{2}\right)\left(n_{2}+\frac{1}{2}\right) \cdots\left(n_{j}+\frac{1}{2}\right) \times \\
& \times e^{-\frac{\hbar \omega_{0}\left(n_{1}+\frac{1}{2}\right)}{K T}} e^{-\frac{\hbar \omega_{0}\left(n_{2}+\frac{1}{2}\right)}{K T}} \cdots e^{-\frac{\hbar \omega_{0}\left(n_{j}+\frac{1}{2}\right)}{K T}}= \\
& =\frac{(2 j) !}{j ! 2^{j}}\left[\frac{\hbar}{2 m \omega_{0}} \operatorname{coth}\left(\frac{\hbar \omega_{0}}{2 K T}\right)\right]^{j}
\end{aligned}
$$

para $j \in\{1,2,3, \ldots\}$. As médias das potências ímpares de $x$ são todas nulas.

Os cálculos realizados para a posição $x$ também podem ser aplicados ao momento $p$. Em uma expressão geral podemos escrever que [14]

$$
\left\langle x^{2 j} p^{2 l}\right\rangle_{T}=\frac{(2 j) !(2 l) !}{j ! l ! 2^{j+l}}\left\langle x^{2}\right\rangle_{T}^{j}\left\langle p^{2}\right\rangle_{T}^{l}
$$

para $j, l \in\{1,2,3, \ldots\}$. As médias de composições com potências ímpares de $x$ ou $p$ são nulas, isto é, $\left\langle x^{j} p^{l}\right\rangle_{T}=0$, sejam $j$ ou $l$ números inteiros ímpares.

Para a QED, a média quadrática da posição $\left\langle\left|\underline{x}^{2}\right|\right\rangle_{T}$ é feita pelas médias dos estados de fótons incoerentes que agem sobre o oscilador. Assim com o auxílio da função de correlação (2.47), temos que

$$
\begin{aligned}
& \left\langle\left|\underline{x}^{2}\right|\right\rangle_{T}=\frac{q^{2}}{m^{2}} \sum_{\alpha_{1}=1}^{2} \sum_{\alpha_{2}=1}^{2} \int d^{3} k_{1} \int d^{3} k_{2} \epsilon_{x_{1}} \epsilon_{x_{2}} \frac{\sqrt{\hbar \omega_{1}}}{2 \pi} \frac{\sqrt{\hbar \omega_{2}}}{2 \pi} \times \\
& \times \sum_{n_{\overrightarrow{k_{1} \alpha_{1}}}=0}^{\infty} \sum_{n_{k_{2} \alpha_{2}}=0}^{\infty} \frac{e^{-\frac{W_{n}\left(\overline{k_{1}}, \alpha_{1}\right)}{K T}}}{\Xi_{1}} \frac{e^{-\frac{W_{n}\left(\overrightarrow{k_{2}, \alpha_{2}}\right)}{K T}}}{\Xi_{2}} \times \\
& \times\left\langle n_{\overrightarrow{k_{1}} \alpha_{1}} n_{\overrightarrow{k_{2}} \alpha_{2}}\right|\left(\frac{a_{1}}{C\left(\omega_{1}\right)} e^{-i \omega_{1} t+i \vec{k}_{1} \cdot \vec{r}_{0}}+\frac{a_{1}^{\dagger}}{C^{*}\left(\omega_{1}\right)} e^{i \omega_{1} t-i \vec{k}_{1} \cdot \vec{r}_{0}}\right) \times \\
& \times\left(\frac{a_{2}}{C\left(\omega_{2}\right)} e^{-i \omega_{2} t+i \vec{k}_{2} \cdot \vec{r}_{0}}+\frac{{\underline{a_{2}}}^{\dagger}}{C^{*}\left(\omega_{2}\right)} e^{i \omega_{2} t-i \vec{k}_{2} \cdot \vec{r}_{0}}\right)\left|n_{\overrightarrow{k_{1} \alpha_{1}}} n_{\overrightarrow{k_{2}} \alpha_{2}}\right\rangle,
\end{aligned}
$$

sendo que os termos $\Xi_{1}$ e $\Xi_{2}$ são as respectivas funções de partição de cada variável de campo da função de correlação (2.47). O autoestado $\left|n_{\overrightarrow{k_{1}} \alpha_{1}} n_{\overrightarrow{k_{2} \alpha_{2}}}\right\rangle$ contem os $n_{\overrightarrow{k_{1} \alpha_{1}}}$ fótons do campo $\underline{E_{i}}\left(\overrightarrow{r_{1}}, t_{1}\right)$ e os $n_{\overrightarrow{k_{2} \alpha_{2}}}$ fótons do campo $\underline{E_{j}}\left(\vec{r}_{2}, t_{2}\right)$. Calculando os valores esperados da expressão (2.72) com o auxílio 
das relações (2.43), obtemos, integrando nos ângulos,

$$
\begin{aligned}
\left\langle\left|\underline{x}^{2}\right|\right\rangle_{T} & =\frac{4 q^{2}}{3 \pi m^{2} c^{3}} \int_{0}^{\infty} \frac{d \omega \omega^{2}}{|C(\omega)|^{2}} \frac{\sum_{n=0}^{\infty} \hbar \omega\left(n+\frac{1}{2}\right) e^{-\frac{\hbar \omega}{K T}\left(n+\frac{1}{2}\right)}}{\sum_{n=0}^{\infty} e^{-\frac{\hbar \omega}{K T}\left(n+\frac{1}{2}\right)}}= \\
& =\frac{2 q^{2}}{3 \pi m^{2} c^{3}} \int_{0}^{\infty} d \omega \frac{\hbar \omega^{3} \operatorname{coth}\left(\frac{\hbar \omega}{2 K T}\right)}{\left(\omega^{2}-\omega_{0}^{2}\right)^{2}+\Gamma^{2} \omega^{6}} .
\end{aligned}
$$

Podemos ver claramente que as expressões (2.68) e (2.73) são idênticas. De fato as médias da posição ou do momento para várias ordens de potências na SED são iguais a suas correspondentes na QED, isto é, $\left\langle x^{j}\right\rangle=\left\langle\underline{x}^{j}\right\rangle$ e $\left\langle p^{j}\right\rangle=\left\langle\underline{p}^{j}\right\rangle$, para um número inteiro positivo $j$ (veja [14]). É evidente que as considerações e aproximações feitas para as expressões (2.68) e (2.69) também valem para a expressão (2.73).

As composições das variáveis $\underline{x}$ e $\underline{p}$ têm em geral valores médios diferentes daqueles das variáveis da SED. Porém devemos notar que, assim como no cálculo das funções de correlação dos campos eletromagnéticos livres, composições de operadores quânticos, que não comutam, podem ser correspondidos com as variáveis clássicas através da correspondência de Weyl [24]. Desse modo o ordenamento simétrico de operadores compostos de $\underline{x}$ e $\underline{p}$ possuem valores médios iguais aos valores médios de suas correspondentes variáveis clássicas. Tal afirmação tem sua prova completa na referência [14].

Vamos, então, discutir as semelhanças entre a SED e a QED, se considerarmos o limite $\Gamma \omega_{0} \ll 1$. Assim vamos descrever explicitamente a conexão entre as duas teorias, não só para osciladores dipolares, mas também para osciladores harmônicos neutros. Por exemplo, alguns valores médios da posição e do momento pela SED são

$$
\begin{gathered}
\langle x\rangle_{T}=0 \quad, \quad\langle p\rangle_{T}=0, \\
\langle x p\rangle_{T}=0, \\
\left\langle x^{2}\right\rangle_{T}=\frac{\hbar}{2 m \omega_{0}} \operatorname{coth}\left(\frac{\hbar \omega_{0}}{2 k T}\right), \\
\left\langle p^{2}\right\rangle_{T}=\frac{\hbar m \omega_{0}}{2} \operatorname{coth}\left(\frac{\hbar \omega_{0}}{2 k T}\right),
\end{gathered}
$$

e pela QED são

$$
\begin{gathered}
\langle|\underline{x}|\rangle_{T}=0 \quad, \quad\langle|\underline{p}|\rangle_{T}=0 \\
\langle|\underline{x} \underline{p}|\rangle_{T}=\frac{i \hbar}{2}=-\langle|\underline{p} \underline{x}|\rangle_{T}
\end{gathered}
$$




$$
\begin{aligned}
\left\langle\left|\underline{x}^{2}\right|\right\rangle_{T} & =\frac{\hbar}{2 m \omega_{0}} \operatorname{coth}\left(\frac{\hbar \omega_{0}}{2 k T}\right), \\
\left\langle\left|\underline{p^{2}}\right|\right\rangle_{T} & =\frac{\hbar m \omega_{0}}{2} \operatorname{coth}\left(\frac{\hbar \omega_{0}}{2 k T}\right) .
\end{aligned}
$$

Cada relação em (2.74) tem o mesmo resultado de sua respectiva relação em (2.75), efetuado o ordenamento simétrico dos operadores quânticos.

Tanto pelas relações (2.74) da SED, quanto pelas relações (2.75) da QED, pode-se calcular a energia média do oscilador harmônico a uma temperatura $T$. Visto que a energia total é dado pelo hamiltoniano $H$, então

$$
\langle\underline{H}\rangle=\langle H\rangle=\frac{1}{2 m}\left\langle p^{2}\right\rangle+\frac{m \omega_{0}^{2}}{2}\left\langle x^{2}\right\rangle=\frac{\hbar \omega_{0}}{2} \operatorname{coth}\left(\frac{\hbar \omega_{0}}{2 K T}\right) .
$$

Conclui-se que, feito o ordenamento simétrico dos operadores quânticos, todos os valores médios da QED são iguais aos respectivos valores médios da SED para o caso do oscilador elétrico dipolar, em particular também para o oscilador harmônico. O que torna as duas teorias equivalentes na descrição estatística desses sistemas físicos. 


\section{Capítulo 3}

\section{O Ruído de Nyquist}

Em 1928, Johnson [19] observou experimentamente que uma tensão elétrica aleatória está sempre presente em condutores em equilíbrio com um reservatório de temperatura não nula. Imediatamente após o trabalho de Johnson, Nyquist apresentou a explicação teórica [20], que consiste em deduzir a tensão elétrica aleatória considerando o condutor em equilíbrio termodinâmico com o meio. Este fenômeno é conhecido por ruído de Johnson-Nyquist, ou apenas de ruído de Nyquist. Com os trabalhos de Callen e Welton [21] e de Callen e Greene [22], foram feitas generalizações da relação de Nyquist, chamadas de teorema flutuação-dissipação. Nesses trabalhos é demonstrada a existência de ruídos para todo sistema físico regido por uma equação linear, estabelecendo uma relação entre a resistência generalizada do sistema com a força flutuante generalizada atuante no sistema. O teorema flutuação-dissipação pode ser aplicado tanto para sistemas quânticos (veja [21]), como para sistemas clássicos (veja [22]).

$\mathrm{Na}$ primeira parte deste capítulo, vamos apresentar a equação de Langevin de um circuito RLC série e algumas de suas propriedades. Posteriormente é apresentada uma dedução do ruído de Nyquist seguindo a análise de Ginzburg [23]. É feita, então, uma breve discussão sobre o teorema flutuação-dissipação.

\subsection{Equação de Langevin}

Existem vários métodos para se estudar sistemas estocásticos, dentre os quais vamos utilizar o que é chamado de equação de Langevin [18]. A equação 
de Langevin é uma equação fenomenológica, para cuja construção toma-se a equação de Newton do sistema, no caso em que não há flutuações, e adicionase um termo dissipativo e um termo de força aleatória $[18,24,28]$. Esse método fornece um caminho para a demonstração da relação de Nyquist e, portanto, para o teorema flutuação-dissipação [23]. A equação de Langevin é originalmente clássica, servindo adequadamente à SED.

É possível escrever-se a equação de Langevin de um circuito RLC série sem baterias, cuja resistência é $R$, capacitância é $C$ e indutância é $L$. Para tal deve-se notar que este sistema físico é análogo a um oscilador harmônico amortecido. A equação procurada deve ter como variáveis dinâmicas aleatórias a carga elétrica no capacitor, $q(t)$, e a tensão elétrica aleatória atuando no sistema, $\varepsilon(t)$, ambas dependentes do tempo. Logo podemos escrever que

$$
L \ddot{q}(t)+R \dot{q}(t)+\frac{1}{C} q(t)=\varepsilon(t) .
$$

É razoável supor que a equação (3.1) é um processo gaussiano, isto é, as distribuições de probabilidades das variáveis estocásticas são completamente determinadas por suas médias

$$
\begin{aligned}
& \langle q(t)\rangle=0, \\
& \langle\varepsilon(t)\rangle=0
\end{aligned}
$$

e suas respectivas médias quadráticas, $\left\langle\varepsilon^{2}(t)\right\rangle$ e $\left\langle q^{2}(t)\right\rangle$, efetuadas sobre um ensemble.

Um caso mais geral da equação (3.1) é obtido quando a força dissipativa num dado instante é dependente dos tempos passados. Para esse caso deve-se considerar que a resistência é uma função dependente do tempo que vamos definir como $\gamma(t)$. Assim a equação do circuito RLC fica $[25,26]$

$$
L \ddot{q}(t)+\int_{t_{0}}^{t} \gamma\left(t-t^{\prime}\right) \dot{q}\left(t^{\prime}\right) d t^{\prime}+\frac{1}{C} q(t)=\varepsilon(t) .
$$

A dissipação de um sistema físico só pode ocorrer após o instante em que ocorre uma perturbação inicial, logo a resistência $\gamma(t)$ é não nula apenas para $t>0$.

Podemos definir a corrente elétrica ao longo do circuito como $I(t)=\dot{q}(t)$. Logo a equação (3.3) torna-se

$$
L \dot{I}(t)+\int_{t_{0}}^{t} \gamma\left(t-t^{\prime}\right) I\left(t^{\prime}\right) d t^{\prime}+\frac{1}{C} \int_{t_{0}}^{t} I\left(t^{\prime}\right) d t^{\prime}=\varepsilon(t)
$$


e consequentemente $\langle I(t)\rangle=0$. A condição de causalidade

$$
\left\langle I\left(t_{1}\right) \varepsilon\left(t_{2}\right)\right\rangle=0, \text { para } t_{2}>t_{1}>t_{0},
$$

impõe-se, pois uma perturbação da tensão em algum instante não deve provocar uma reação do circuito em tempos passados.

Devemos notar que o termo dissipativo é uma função linear com relação à corrente $I(t)$, o que significa que o sistema dissipativo (circuito RLC) está acoplado linearmente com o reservatório (banho térmico). Esse é um caso particular de dissipação, no qual o circuito RLC está fracamente acoplado a cada modo flutuante do banho térmico. No entanto, quando somados todos os acoplamentos do circuito com cada modo de oscilação do banho térmico, a dissipação total pode ser muito grande [24].

Para que as equações dinâmicas (3.1), (3.3) e (3.4) sejam válidas, é necessário considerar que as dimensões do circuito são muito menores que os comprimentos de onda das oscilações presentes no circuito. Então deve-se usar a aproximação de comprimentos de onda longos, que é consistente com uma abordagem não relativística. Assim dado um comprimento típico do circuito, $l$, então os comprimentos de onda das flutuações dos campos em torno do circuito, $\lambda$, seguem que

$$
l \ll \lambda \equiv \frac{2 \pi c}{\omega} .
$$

Sob essas condições, os campos e correntes no circuito seguem a aproximação quase-estacionária, em que a corrente $I(t)$, em um dado instante $t$, é igual em todo o circuito.

Os transientes do circuito não são de interesse, pois não contribuem para os valores médios e funções de correlação analisados neste capítulo. Assim vamos estudar apenas o estado estacionário do circuito, que pode ser convenientemente obtido pela análise de Fourier. As transformadas de Fourier das variáveis estocásticas $I(t)$ e $\varepsilon(t)$ são

$$
\tilde{I}(\omega)=\frac{1}{2 \pi} \int_{-\infty}^{\infty} I(t) e^{-i \omega t} d t
$$

e

$$
\tilde{\varepsilon}(\omega)=\frac{1}{2 \pi} \int_{-\infty}^{\infty} \varepsilon(t) e^{-i \omega t} d t .
$$


Aplicando as expressões (3.7) e (3.8) na equação do circuito (3.4) é possível obter que

$$
\tilde{I}(\omega)=\frac{1}{\tilde{\gamma}(\omega)+i\left(\omega L-\frac{1}{\omega C}\right)} \tilde{\varepsilon}(\omega)
$$

sendo que $\tilde{\gamma}(\omega)$ é a transformada de Fourier-Laplace da função de dissipação $\gamma(t)$, isto é,

$$
\tilde{\gamma}(\omega)=\int_{0}^{\infty} \gamma(t) e^{-i \omega t} d t
$$

Dessa forma a transformada de Fourier da força eletromotriz dissipativa de (3.4) é calculada pela convolução

$$
\frac{1}{2 \pi} \int_{-\infty}^{\infty} d t \int_{t_{0}}^{t} d t^{\prime} \gamma\left(t-t^{\prime}\right) I\left(t^{\prime}\right) e^{-i \omega t}=\tilde{\gamma}(\omega) \tilde{I}(\omega)
$$

Da maneira como foi introduzido o termo dissipativo na equação (3.4), a função $\gamma(t)$ e sua correspondente função transformada $\tilde{\gamma}(\omega)$ têm não só uma componente dissipativa, mas também uma componente difusiva. Isso pode ser verificado notando que a função $\tilde{\gamma}(\omega)$ é complexa, cuja parte real corresponde a um fator dissipativo (no caso de circuitos elétricos, resistivo), e cuja parte imaginária corresponde a um fator difusivo (no caso de circuitos elétricos, reativo), conforme as discussões feitas por Kubo [25, 26], Gardiner [27] e Landau [29]. Contudo, dentro do escopo desta dissertação, vamos considerar que toda contribuição reativa do circuito elétrico deve-se apenas à capacitância $C$ e à indutância $L$. Para tal é necessário substituir a transformação (3.10) por

$$
\Re e \tilde{\gamma}(\omega)=\frac{1}{2} \int_{-\infty}^{\infty} \gamma(t) e^{-i \omega t} d t
$$

A parte real de $\tilde{\gamma}(\omega)$ corresponde à resistência elétrica para cada modo de oscilação de freqüência $\omega$, assim vamos definir que $R(\omega) \equiv \mathfrak{R e} \tilde{\gamma}(\omega)$. A expressão (3.12) pode ser invertida, de modo que

$$
\gamma(t)=\frac{1}{\pi} \int_{-\infty}^{\infty} \Re(\tilde{\gamma}(\omega)) e^{i \omega t} d \omega .
$$

Nota-se que a função $\gamma(t)$ foi estendida para valores negativos de $t$, de modo que $\gamma(t)=\gamma(-t)$. Isso pode ser contornado multiplicando $\gamma(t)$ por uma função degrau [27]. 
Com essas considerações, podemos usar um termo puramente resistivo, $R(\omega)$. Assim a solução transformada de Fourier da equação (3.4) é

$$
\tilde{I}(\omega)=\frac{\tilde{\varepsilon}(\omega)}{Z(\omega)}
$$

tal que $Z(\omega)$ é a impedância do circuito elétrico, ou seja,

$$
Z(\omega)=R(\omega)+i\left(\omega L-\frac{1}{\omega C}\right)
$$

A distribuição espectral da corrente flutuante, $S_{I}(\omega)$, está relacionada com a função de correlação temporal dessa mesma corrente, $\left\langle I\left(t_{0}\right) I\left(t_{0}+t\right)\right\rangle$, através das relações de Wiener-Khintchine (veja $[26,28])$, que se constituem, no caso da corrente elétrica, no seguinte par de equações,

$$
\begin{aligned}
& S_{I}(\omega)=\frac{1}{2 \pi} \int_{-\infty}^{\infty}\left\langle I\left(t_{0}\right) I\left(t_{0}+t\right)\right\rangle e^{-i \omega t} d t, \\
& \left\langle I\left(t_{0}\right) I\left(t_{0}+t\right)\right\rangle=\int_{-\infty}^{\infty} S_{I}(\omega) e^{i \omega t} d \omega .
\end{aligned}
$$

No caso da tensão elétrica flutuante, as relações de Wiener-Khintchine são análogas às equações (3.16), apenas notando que a distribuição espectral deve ser $S_{\varepsilon}(\omega)$ e a função de correlação temporal, $\left\langle\varepsilon\left(t_{0}\right) \varepsilon\left(t_{0}+t\right)\right\rangle$.

Como a corrente e a tensão estão no estado estacionário, é possível efetuar as transformações de Fourier sobre suas funções de correlação temporal. Com o uso das relações de Wiener-Khintchine, obtemos as respectivas funções de correlação dependentes das freqüências, ou seja,

$$
\left\langle\tilde{I}(\omega) \tilde{I}\left(\omega^{\prime}\right)\right\rangle=S_{I}(\omega) \delta\left(\omega+\omega^{\prime}\right)
$$

e

$$
\left\langle\tilde{\varepsilon}(\omega) \tilde{\varepsilon}\left(\omega^{\prime}\right)\right\rangle=S_{\varepsilon}(\omega) \delta\left(\omega+\omega^{\prime}\right) .
$$

Como a corrente e a tensão transformadas estão relacionadas por (3.14), então as distribuições espectrais são vinculadas por

$$
S_{I}(\omega)=\frac{S_{\varepsilon}(\omega)}{|Z(\omega)|^{2}} .
$$

A determinação das funções de correlação (3.17) e (3.18) é apresentada na seção a seguir. 


\subsection{Ruído de Nyquist}

As flutuações de tensão e corrente no circuito elétrico são causadas pela agitação térmica dos elétrons contidos nos condutores. Assim, segundo $\mathrm{Ny}$ quist [20], para se determinar o comportamento flutuante das tensões e correntes, é necessário considerar um circuito em equilíbrio termodinâmico, à uma temperatura $T$. Tais considerações também são feitas por Ginzburg [23]. Além disso, como fez Ginzburg, pode-se tomar um circuito elétrico linear RLC série sem baterias, como já foi considerado anteriormente. As dimensões do circuito devem ser muito menores que o comprimento de onda das oscilações do circuito, valendo a condição (3.6), de modo que a corrente no circuito segue a aproximação quase-estacionária. Então o comportamento do circuito deve seguir as expressões discutidas na seção anterior.

Podemos considerar que um circuito pode ser particionado arbitrariamente em outros dois circuitos de características distintas ( $R, L$ e $C$ distintos), ligados em série. Vamos indicar uma parte pelo índice 1 e a outra pelo índice 2. Como já foi levado em conta, a corrente $I(t)$ é a mesma em todo o circuito, logo as tensões em cada parte do circuito são respectivamente $\varepsilon_{1}=Z_{1} I$ e $\varepsilon_{2}=Z_{2} I$, sendo $Z_{1}$ e $Z_{2}$ as impedâncias e $R_{1}$ e $R_{2}$ as resistências de cada respectivo circuito. No equilíbrio termodinâmico, as potências médias transferidas de um circuito para o outro devem ser iguais, isto é, a potência média dissipada em $Z_{1}$ devida a $\varepsilon_{2}\left(P_{1-2}\right)$ é igual a potência média dissipada em $Z_{2}$ devida a $\varepsilon_{1}\left(P_{2-1}\right)$. Lembrando de que

$$
P=\langle I \varepsilon\rangle=\left\langle R I^{2}\right\rangle=\int_{0}^{\infty} R(\omega) S_{I}(\omega) d \omega
$$

e utilizando a equação (3.19), temos então

$$
P_{1-2}=\int_{0}^{\infty} \frac{R_{1} S_{\varepsilon_{2}}(\omega)}{|Z(\omega)|^{2}} d \omega=P_{2-1}=\int_{0}^{\infty} \frac{R_{2} S_{\varepsilon_{1}}(\omega)}{|Z(\omega)|^{2}} d \omega
$$

sendo que $Z(\omega)=Z_{1}(\omega)+Z_{2}(\omega)$. A equação (3.21) não só deve ser satisfeita com $\omega$ variando de 0 a $\infty$, mas também para qualquer variação entre $\omega$ e $\omega+\Delta \omega$. Isto segue do princípio de balanço detalhado, ou seja, no equilíbrio termodinâmico as potências transferidas entre as partes do circuito são iguais, independentemente do intervalo de freqüência $\Delta \omega$ da corrente flutuante [23, 28 ]. Isto só pode ser satisfeito quando os integrandos de (3.21) forem iguais, logo

$$
R_{1} S_{\varepsilon_{2}}(\omega)=R_{2} S_{\varepsilon_{1}}(\omega)
$$


Reescrevendo a equação (3.22), obtem-se a seguinte expressão geral

$$
\frac{S_{\varepsilon_{1}}(\omega)}{R_{1}}=\cdot \frac{S_{\varepsilon_{2}}(\omega)}{R_{2}}=\frac{S_{\varepsilon}(\omega)}{R}=f(\omega, T)
$$

A função $f(\omega, T)$ é universal e depende apenas de $\omega$ e da temperatura $T$, pois as partes consideradas do circuito são arbitrárias. Logo a função $f(\omega, T)$ não deve depender de $L, C$, e $R$, e para sua determinação é necessário calcular a energia média total armazenada no circuito. A energia média do campo elétrico é dada por

$$
\begin{aligned}
\langle U\rangle & =\left\langle\frac{q^{2}}{2 C}\right\rangle=\frac{1}{2 C} \int_{-\infty}^{\infty} \frac{S_{I}(\omega)}{\omega^{2}} d \omega=\frac{1}{C} \int_{0}^{\infty} \frac{S_{\varepsilon}(\omega)}{\omega^{2}|Z(\omega)|^{2}} d \omega= \\
& =\int_{0}^{\infty} \frac{C R f(\omega, T)}{R^{2} C^{2} \omega^{2}+\left(L C \omega^{2}-1\right)^{2}} d \omega .
\end{aligned}
$$

E a média da energia do campo magnético armazenada é

$$
\begin{aligned}
\langle Q\rangle & =\left\langle\frac{L I^{2}}{2}\right\rangle=\frac{L}{2} \int_{-\infty}^{\infty} S_{I}(\omega) d \omega= \\
& =\int_{0}^{\infty} \frac{\omega^{2} C^{2} L R f(\omega, T)}{R^{2} C^{2} \omega^{2}+\left(L C \omega^{2}-1\right)^{2}} d \omega .
\end{aligned}
$$

Para um valor pequeno de $R$, notamos que as integrais (3.24) e (3.25) possuem um pico muito acentuado em $\omega=(L C)^{-\frac{1}{2}}$. Logo as maiores contribuições para as integrais vêm da região em torno dos picos. Assim aproximando o termo $f(\omega, T)$ das integrais como uma função constante nessa região e integrando por partes, temos

$$
\begin{aligned}
\langle U\rangle & =f(\Omega, T) \int_{0}^{\infty} \frac{\alpha d \zeta}{\alpha^{2} \zeta^{2}+\left(\zeta^{2}-1\right)^{2}}= \\
\langle Q\rangle & =f(\Omega, T) \int_{0}^{\infty} \frac{\alpha \zeta^{2} d \zeta}{\alpha^{2} \zeta^{2}+\left(\zeta^{2}-1\right)^{2}}= \\
& =\frac{\pi}{2} f(\Omega, T),
\end{aligned}
$$

para $\alpha=\frac{C R}{\sqrt{L C}}, \Omega=\frac{1}{\sqrt{L C}}$ e $\zeta=\frac{\omega}{\Omega}$.

Por outro lado, para $R$ tendendo a zero, a equação do circuito (3.1) ou (3.3) tende à equação do oscilador harmônico

$$
L \ddot{q}(t)+\frac{q(t)}{C}=\varepsilon(t)
$$


Como já foi discutido no capítulo anterior, o comportamento do oscilador harmôntica code ser analisado de maneira consistente tanto pela Mecânica médios das variáva Eletrodinâmica Estocástica, obtendo os mesmos valores média do circuito é

$$
\langle W\rangle=\langle U\rangle+\langle Q\rangle=\left\langle\frac{q^{2}}{2 C}\right\rangle+\left\langle\frac{L I^{2}}{2}\right\rangle=\frac{\hbar \Omega}{2} \operatorname{coth}\left(\frac{\hbar \Omega}{2 K T}\right) .
$$

Como a equação (3.27) pode descrever partes arbitrárias do circuito com características $L$ e $C$ arbitrárias, então $\Omega$ é também arbitrária. Assim tomando as equações (3.26) e (3.28), obtem-se

$$
f(\omega, T)=\frac{\hbar \omega}{2 \pi} \operatorname{coth}\left(\frac{\hbar \omega}{2 K T}\right) .
$$

Substituindo (3.29) na relação (3.23), então a distribuição espectral da tensão é

$$
S_{\varepsilon}(\omega)=\frac{\hbar \omega R(\omega)}{2 \pi} \operatorname{coth}\left(\frac{\hbar \omega}{2 K T}\right) .
$$

Substituindo a expressão (3.30) em (3.18), obtemos, então a relação de Nyquist para a tensão elétrica flutuante

$$
\left\langle\tilde{\varepsilon}(\omega) \tilde{\varepsilon}\left(\omega^{\prime}\right)\right\rangle=R(\omega) \frac{\hbar \omega}{2 \pi} \operatorname{coth}\left(\frac{\hbar \omega}{2 K T}\right) \delta\left(\omega+\omega^{\prime}\right) .
$$

Usando a relação (3.19), obtemos a relação de Nyquist para a corrente flutuante

$$
\left\langle\tilde{I}(\omega) \tilde{I}\left(\omega^{\prime}\right)\right\rangle=\frac{R(\omega)}{|Z(\omega)|^{2}} \frac{\hbar \omega}{2 \pi} \operatorname{coth}\left(\frac{\hbar \omega}{2 K T}\right) \delta\left(\omega+\omega^{\prime}\right) .
$$

As relações (3.31) e (3.32) podem ser facilmente generalizadas na forma do teorema flutuação-dissipação, que são relações gerais entre a impedância generalizada de um sistema linear e a força flutuante generalizada atuando sobre esse sistema. Embora as relações obtidas aqui sejam um caso particular do teorema flutuação-dissipação, elas lhe são completamente análogas, se especificados claramente os significados das variáveis [23, 29]. A relevância do teorema flutuação-dissipação está na demonstração da ampla aplicabilidade das relações (3.31) e (3.32), incluindo os sistemas lineares quânticos e clássicos. 
A dedução feita por Ginzburg [23] tem a vantagem de mostrar explicitamente a condição de resistência pequena, $R(\omega) \rightarrow 0$, de modo que sejam válidas as equações (3.27) e (3.28). Um outro limite que pode ser considerado é $\frac{R(\omega)}{L \Omega} \ll 1$, visto que para este caso a freqüência natural do circuito tende a ser $\Omega$, de modo que a expressão (3.28) é válida. Esses limites apresentados se aplicam a circuitos lineares em geral, como foi citado na seção anterior. Os sistemas físicos que não seguem estes limites levam a complicações no tratamento quântico, assim como na SED, que incluem comportamentos não-lineares, e não cabem discuti-las nesta dissertação. 


\section{Capítulo 4}

\section{Interação do solenóide com o oscilador dipolar elétrico}

Estudos sobre a interação dos campos eletromagnéticos do vácuo com a matéria têm sido feitos desde as origens da Eletrodinâmica Quântica. Várias descobertas teóricas de fenômenos envolvendo a radiação do vácuo foram feitas, inclusive efeitos sobre objetos macroscópicos, dentre os quais podemos citar o efeito Casimir [12].

Outros efeitos importantes da radiação do vácuo são as alterações da taxa de emissão espontânea de transições atômicas, quando os átomos interagem com circuitos ressonantes ou paredes condutoras ou dielétricas. Tais efeitos devem-se a mudanças que a radiação do vácuo sofre com ambientes diferentes do espaço livre, consequentemente provocando alterações nos átomos. Essa área da Eletrodinâmica Quântica é hoje conhecida como Eletrodinâmica de Cavidades.

Há alguns anos, foi feito um trabalho por França e Marshall, em que se estuda o comportamento de átomos excitados entre espelhos [31], usando o formalismo da Eletrodinâmica Estocástica. Os resultados obtidos por França e Marshall [31] estão em acordo com os resultados experimentais obtidos por Jhe et al. [32]. Isso motivou vários outros trabalhos, em que se utiliza a SED para se estudar a interação de osciladores dipolares elétricos microscópicos com um solenóide macróscopico, feitos por Blanco, Dechoum, França, Santos e Maia Jr. $[1,2,3,6]$. Neste capítulo apresentamos os desenvolvimentos feitos por essas referências. 


\subsection{Descrição do sistema físico}

O sistema físico analisado por Blanco, Dechoum, França, Santos e Maia Jr. $[1,2,3]$ é constituído de um solenóide macroscópico ligado a um circuito RLC série sem baterias. Próximo do solenóide há um dipolo elétrico oscilante microscópico. O tratamento utilizado é não relativístico.

Por simplificação, sem perder a generalidade do problema, o solenóide considerado é cilíndrico, com comprimento $l$ e raio $a$, feito de $N$ espiras circulares. $\mathrm{O}$ oscilador elétrico encontra-se na origem de um sitema de coordenadas cartesiano, tal que o movimento do oscilador é unidimesional e orientado na direção do eixo $0-x$. A uma distância $y$ do oscilador, na direção $0-y$, está o centro do solenóide, cujo eixo de simetria é paralelo à direção $0-z$. Todo esse arranjo está imerso em um banho de radiação térmica e de ponto-zero. A figura 4.1 mostra uma representação esquemática do sistema.

\section{EIXO DO SOLENÓIDE}

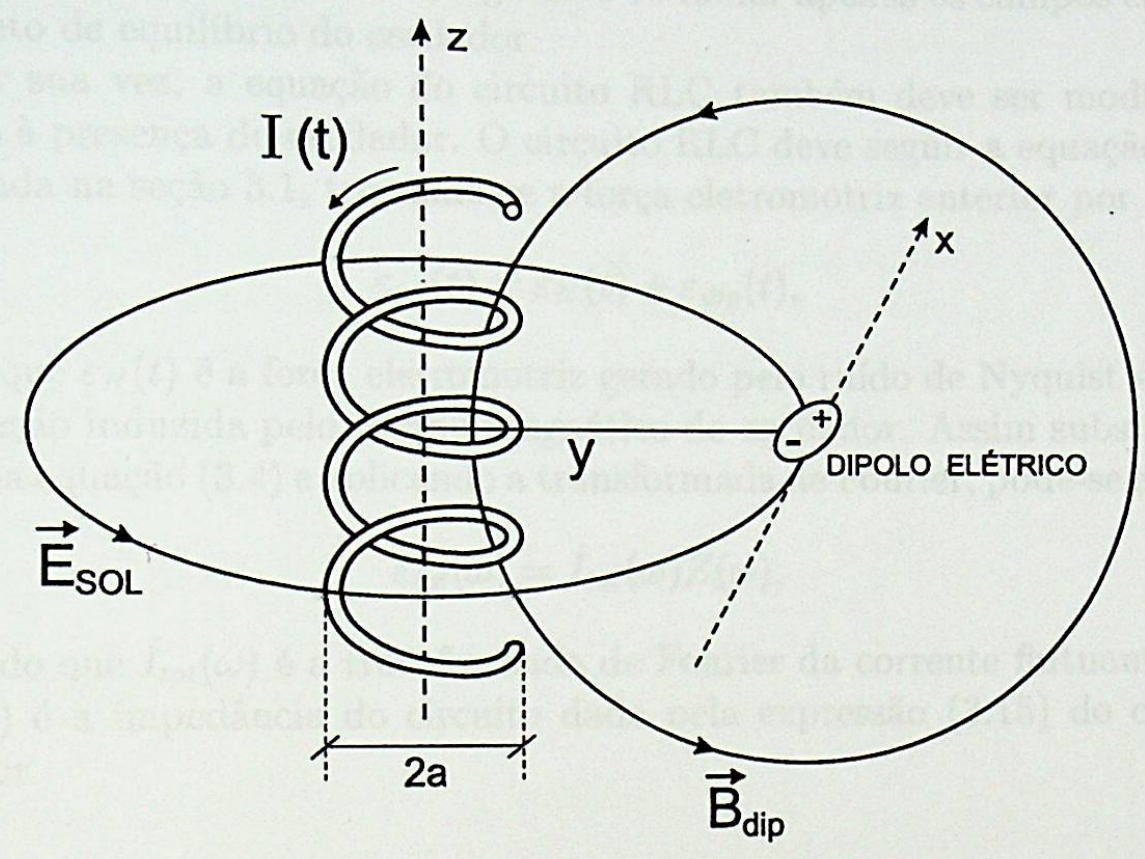

Figura 4.1: Figura esquemática do dipolo elétrico a uma distância $y$ do eixo do solenóide. Os campos relevantes gerados pelo solenóide $\vec{E}_{\text {sol }}$ e pelo dipolo oscilante $\vec{B}_{d i p}$ estão indicados 
Assim como foi discutido na seção 2.3, o oscilador elétrico tem massa $m$ (2.62)), mas o campo elétrico resultante devido a presença do solenóide (ver
figura 4.1). figura 4.1). Então a equação do movimento do oscilador elétrico é

$$
m \ddot{x}=-m \omega_{0}^{2} x+\frac{2 q^{2}}{3 c^{3}} \dddot{x}+q E_{t o t}(t),
$$

sendo que o campo total agindo no oscilador é a soma da componente na direção $0-x$ do campo elétrico livre, $E_{x}(t)$, mais o campo elétrico produzido pelo solenóide, $E_{\text {sol }}(t)$, isto é,

$$
E_{t o t}(t)=E_{x}(t)+E_{\text {sol }}(t)
$$

Os campos que agem no oscilador dependem apenas do tempo, porque o tratamento é não relativístico, logo deve-se tomar apenas os campos elétricos no ponto de equilíbrio do oscilador.

Por sua vez, a equação do circuito RLC também deve ser modificada, devido à presença do oscilador. O circuito RLC deve seguir a equação (3.4), explicada na seção 3.1, trocando-se a força eletromotriz anterior por

$$
\varepsilon_{t o t}(t)=\varepsilon_{N}(t)+\varepsilon_{d i p}(t),
$$

sendo que $\varepsilon_{N}(t)$ é a força eletromotriz gerado pelo ruído de Nyquist e $\varepsilon_{\text {dip }}(t)$ é a tensão induzida pelo campo magnético do oscilador. Assim substituindo (4.3) na equação (3.4) e aplicando a transformada de Fourier, pode-se escrever

$$
\tilde{\varepsilon}_{t o t}(\omega)=\tilde{I}_{t o t}(\omega) Z(\omega),
$$

de modo que $\tilde{I}_{\text {tot }}(\omega)$ é a transformada de Fourier da corrente flutuante total e $Z(\omega)$ é a impedância do circuito dada pela expressão (3.15) do capítulo anterior.

\subsection{Flutuações do sistema e equações da in- teração}

Além das equações que regem o circuito elétrico e o oscilador dipolar, já podemos determinar as propriedades estatísticas de algumas variáveis. Como 
estamos usando o formalismo da SED, a função de correlação do campo elétrico do vácuo é dada pela expressão (2.35). No limite de comprimentos
de onda longos, a expressão $(2.35)$ reduz-se a

$$
\left\langle E_{i}\left(t_{1}\right) E_{j}\left(t_{2}\right)\right\rangle_{T}=\int_{-\infty}^{\infty} d \omega \delta_{i j} \frac{\hbar \omega^{3}}{3 \pi c^{3}} \operatorname{coth}\left(\frac{\hbar \omega}{2 K T}\right) e^{i \omega\left(t_{1}-t_{2}\right)}
$$

A componente na direção $x$ do campo elétrico livre é a unica que age sobre o oscilador. Logo aplicando as relações de Wiener-Khintchine, a função de correlação da transformada de Fourier do campo elétrico livre é

$$
\left\langle\tilde{E}_{x}(\omega) \tilde{E}_{x}\left(\omega^{\prime}\right)\right\rangle=\frac{\hbar \omega^{3}}{3 \pi c^{3}} \operatorname{coth}\left(\frac{\hbar \omega}{2 K T}\right) \delta\left(\omega+\omega^{\prime}\right) .
$$
Devemos lembrar que a função de correlação da tensão gerada pelo ruído
de Nyquist é

$$
\left\langle\tilde{\varepsilon}_{N}(\omega) \tilde{\varepsilon}_{N}\left(\omega^{\prime}\right)\right\rangle=\frac{R(\omega) \hbar \omega}{2 \pi} \operatorname{coth}\left(\frac{\hbar \omega}{2 K T}\right) \delta\left(\omega+\omega^{\prime}\right),
$$

sendo que $R(\omega)$ é a resistência ôhmica total do circuito.

A interação entre o solenóide e o oscilador dipolar ocorre através dos campos eletromagnéticos gerados por eles. $\mathrm{O}$ oscilador elétrico sofre a ação da componente $x$ do campo elétrico gerado pelo solenóide, $E_{\text {sol }}$. A figura 1 representa este campo sobre oscilador. Na aproximação de comprimentos de onda longos, deve-se calcular o campo elétrico apenas na posição de equilíbrio do oscilador, então

$$
E_{\text {sol }}(t)=-\frac{1}{c} \frac{\partial A}{\partial t}=\int_{-\infty}^{\infty} d \omega i \omega \tilde{I}_{t o t}(\omega) \frac{b(y, \omega)}{c^{2}} e^{i \omega t}
$$

tal que (veja o apêndice A)

$$
\begin{aligned}
b(y, \omega) & =\frac{\pi N a^{2} y}{l} \int_{-\frac{l}{2}}^{\frac{l}{2}} d z e^{\frac{i \omega}{c} \sqrt{z^{2}+y^{2}}}\left[\frac{1}{\left(z^{2}+y^{2}\right)^{\frac{3}{2}}}-\frac{i \omega}{c\left(z^{2}+y^{2}\right)}\right] \\
& \simeq \frac{2 \pi N a^{2}}{y \sqrt{l^{2}+4 y^{2}}} .
\end{aligned}
$$

O fator $b(y, \omega)$ depende da geometria do solenóide, nesse caso foi considerado um solenóide retilíneo composto por espiras paralelas uniformemente 
distribuídas de $-l / 2$ a $l / 2$. Foi considerado que $a<y$, ou seja, o oscilador ao limite de comprime. A aproximação indicada na expressão (4.9) se refere os efeitos de retardo dos de onda longos, $l \ll c / \omega$, em que se desprezam que valha a desigualdade $y$ pos. No caso de um solenóide muito longo, em

Assim, substituindo $y \ll l$, tem-se que $b(y, \omega)=\frac{2 \pi N a^{2}}{y l}$.

solenóide agindo sobro (4.9) em (4.8), obtem-se o campo elétrico gerado pelo sobre o oscilador elétrico,

$$
E_{\text {sol }}(t) \simeq-\frac{b(y, \omega)}{c^{2}} \dot{I}_{t o t}(t)
$$

sendo que $I_{\text {tot }}(t)$ é a corrente flutuante total no circuito.

A distribuição espectral do campo elétrico resultante, $E_{t o t}$, é diferente da distribuição espectral do campo elétrico livre, devido à presença do solenóide. Para verificarmos isso, vamos considerar o caso em que não há interação com o oscilador. Assim, a partir das expressões (4.2), (4.6), (4.7) e (4.10), a função de correlação de $\tilde{E}_{t o t}(\omega)$ é

$$
\left\langle\tilde{E}_{t o t}(\omega) \tilde{E}_{t o t}\left(\omega^{\prime}\right)\right\rangle=\frac{\hbar \omega^{3}}{3 \pi c^{3}} \operatorname{coth}\left(\frac{\hbar \omega}{2 k T}\right)[1+\beta(\omega, y)] \delta\left(\omega+\omega^{\prime}\right),
$$

na qual foi definida a função

$$
\beta(\omega, y) \equiv \frac{3 R(\omega) b^{2}(y)}{2 c|Z(\omega)|^{2}}
$$

sendo que $b(y)$ é dado por (4.9). Estamos supondo que os campos com origens distintas, o campo gerado pelo ruído de Nyquist no solenóide, $E_{\text {sol }}, \mathrm{e}$ o campo do vácuo, $E_{x}$, não são correlacionados, isto é, $\left\langle\tilde{E}_{x}(\omega) \tilde{E}_{s o l}(\omega)\right\rangle=0$. A função de correlação (4.11) mostra que a presença do solenóide altera a distribuição espectral dos campos eletromagnéticos do vácuo. Deve-se notar que tal mudança é significativa para as freqüências angulares próximas da freqüência de ressonância do circuito $\Omega=(L C)^{-\frac{1}{2}}$.

O oscilador elétrico dipolar, por sua vez, produz um campo magnético que induz uma força eletromotriz $\varepsilon_{\text {dip }}$ no solenóide (veja a figura 1 ). A componente na direção $z$ do campo magnético gerado pelo oscilador no ponto $(0, y, z)$ é dada por [16]

$$
\left(B_{d i p}\right)_{z}=\frac{q y \dot{x}(t-r / c)}{c r^{3}}+\frac{q y \ddot{x}(t-r / c)}{c^{2} r^{2}}
$$


sendo que $r^{2}=y^{2}+z^{2}$ e $x(t)$ é a solução da equação (4.1). A tensão induzida pelo campo magnético $\left(B_{\text {dip }}\right)_{z}$ pode ser calculada (veja o apêndice A) e seu
resultado é

$$
\varepsilon_{d i p}(t)=-\frac{1}{c} \frac{d \varphi(t)}{d t}=q \int_{-\infty}^{\infty} d \omega(i \omega)^{2} \tilde{x}(\omega) \frac{b(y, \omega)}{c^{2}} e^{i \omega t}
$$

sendo que $\varphi(t)$ é o fluxo do campo magnético do dipolo nas espiras do solenóide. Os efeitos de campos retardados foram desprezados.

A tensão elétrica total no circuito é a soma da tensão do ruído de Nyquist com a tensão induzida pelo oscilador. Como $b(y, \omega)$ é dado por $(4.9)$, então

$$
\varepsilon_{t o t}(t)=\varepsilon_{N}(t)+\frac{q}{c^{2}} b(y, \omega) \ddot{x}(t)
$$

Substituindo a equação (4.10) na equação (4.1) e aplicando a transformada de Fourier, então

$$
\left(\omega_{0}^{2}-\omega^{2}-i \Gamma \omega^{3}\right) \tilde{x}(\omega)=\frac{q}{m}\left[\tilde{E}_{x}(\omega)-\frac{i \omega b(y, \omega)}{c^{2}} \tilde{I}_{t o t}(\omega)\right]
$$

sendo que $\tilde{x}(\omega)$ é a transformada de Fourier de $x(t)$ e $\Gamma=\frac{2 q^{2}}{3 m c^{3}}$. Substituindo a equação (4.15) na expressão (4.4), obtem-se

$$
Z(\omega) \tilde{I}_{t o t}(\omega)=\tilde{\varepsilon}_{N}(\omega)-\frac{q \omega^{2} b(y, \omega) \tilde{x}(\omega)}{c^{2}}
$$

O par de equações acopladas (4.16) e (4.17) têm como incógnitas $\tilde{x}(\omega)$ e $\tilde{I}_{t o t}(\omega)$. Esse sistema de equações pode ser resolvido exatamente e as suas soluções são estudadas nas seções seguintes.

\subsection{Propriedades do dipolo elétrico}

Para se analisar as novas propriedades do oscilador com a presença do solenóide, as equações (4.16) e (4.17) devem ser resolvidas. Assim é possível obter a expressão de $\tilde{x}(\omega)$,

$$
\tilde{x}(\omega)=\left(\frac{q}{m}\right) \frac{\tilde{E}_{F V}(\omega)-\frac{i \omega b(y)}{c^{2} Z(\omega)} \tilde{\varepsilon}_{N}(\omega)}{\omega_{0}-\omega^{2}-i \omega^{3} \Gamma\left(\frac{3 b^{2}(y)}{2 c Z(\omega)}\right)}=\left(\frac{q}{m}\right) \frac{\tilde{E}_{F V}(\omega)-\frac{i \omega b(y)}{c^{2} Z(\omega)} \tilde{\varepsilon}_{N}(\omega)}{D(\omega)}
$$


sendo que é definido

$$
D(\omega) \equiv \omega_{0}^{2}+\Gamma \omega^{3} \Delta(\omega, y)-\omega^{2}-i \Gamma \omega^{3}[1+\beta(\omega, y)],
$$

tal que as funções $\Delta(\omega, y)$ e $\beta(\omega, y)$ são as funções reais da expressão

$$
\beta(\omega, y)+i \Delta(\omega, y)=\frac{3 b^{2}(y)}{2 c Z(\omega)},
$$

o que nos permite definir

$$
\Delta(\omega, y) \equiv \frac{3 b^{2}(y)}{2 c|Z(\omega)|^{2}}\left(\frac{1}{\omega C}-\omega L\right)
$$

É necessário lembrar que os efeitos de campos retardados foram desprezados. Deve-se notar que, na expressão (4.18), o campo elétrico do vácuo $\tilde{E}_{x}(\omega)$ não é correlacionado com o campo elétrico gerado pelo solenóide $\frac{i \omega b(y)}{c^{2} Z(\omega)} \tilde{\varepsilon}_{N}(\omega)$.

No equilíbrio, podemos calcular a média da expressão (4.18). Como $\tilde{x}(\omega)$ depende das variáveis estocásticas $E_{x}$ e $\varepsilon$, e levando em conta as suas propriedades estatísticas, a média de (4.18) é $\langle x\rangle=0$.

A média quadrática de $\tilde{x}(\omega)$, no equilíbrio, deve ser escrita como

$$
\left\langle x^{2}\right\rangle=\frac{2 q^{2}}{m^{2}} \int_{0}^{\infty} d \omega \frac{\frac{\hbar \omega^{3}}{3 \pi c^{3}} \operatorname{coth}\left(\frac{\hbar \omega}{2 K T}\right)[1+\beta(\omega, y)]}{\left(\omega_{0}^{\prime 2}-\omega^{2}\right)^{2}+\Gamma^{2} \omega^{6}[1+\beta(\omega, y)]^{2}}
$$

sendo que a freqüência $\omega_{0}^{\prime}$ é tal que

$$
\left(\frac{\omega_{0}^{\prime}}{\omega_{0}}\right)^{2} \simeq 1+\Gamma \omega_{0} \Delta\left(\omega_{0}, y\right) \simeq 1+0\left(\frac{q^{2}}{\hbar c} \frac{\hbar \omega_{0}}{m c^{2}}\right)
$$

$\operatorname{assim} \omega_{0}^{\prime} \simeq \omega_{0}$, pois $\frac{\hbar \omega_{0}}{m c^{2}} \ll 1 \mathrm{e} \frac{q^{2}}{\hbar c}=\frac{1}{137}$.

Para o cálculo de $\left\langle x^{2}\right\rangle$, é necessário supor que $\Delta(\omega, y)$ e $\beta(\omega, y)$ são funções suaves e devem ser aproximadamente constantes para valores de $\omega$ próximos de $\omega_{0}$. Além disso $\Gamma \omega_{0}<<1$, de modo que, seguindo o procedimento feito pelas referências $[14,15]$, se obtem

$$
\left\langle x^{2}\right\rangle \simeq \frac{\hbar}{2 m \omega_{0}} \operatorname{coth}\left(\frac{\hbar \omega_{0}}{2 K T}\right)
$$


Note que este resultado é aproximado $[1,2,3]$. Um cálculo mais cuidadoso poderia revelar correções radiativas que dependeriam das características do

A expressão (4.24) é essencialmente o valor da média quadrática de $x$ para um oscilador no espaço livre. Na obtenção de (4.24) a partir de (4.22), o fator $[1+\beta(\omega, y)]$ que aparece no numerador do integrado de (4.22) é cancelado pelo mesmo fator $[1+\beta(\omega, y)]$ no denominador. Isso significa que o efeito devido à presença do solenóide sobre o oscilador é compensado pela reação do oscilador a esse efeito. Isto é um resultado notável do comportamento de flutuação-dissipação de sistemas estocásticos na SED.

\subsection{Emissão espontânea}

A excitação e o decaimento de estados excitados do oscilador dipolar elétrico possuem comportamentos diferentes com a presença do solenóide. Como feito pelas referências [2,3], vamos considerar como uma excitação externa determinística a aplicação de um campo elétrico conhecido. Se o oscilador está próximo do solenóide e é aplicado um campo elétrico determinístico da forma $E_{\text {ext }}(t)=\alpha \delta(t)$ na direção $x$, então a expressão (4.18) da posição do oscilador deve ser reescrita como

$$
\tilde{x}(\omega)=\frac{q\left[\tilde{E}_{x}(\omega)+\tilde{E}_{\text {ext }}(\omega)-\frac{i \omega b(y)}{c^{2} Z(\omega)} \tilde{\varepsilon}_{N}(\omega)\right]}{m D(\omega)} .
$$

A média da posição do oscilador após a excitação do campo $E_{\text {ext }}(t)$ é

$$
\begin{aligned}
\langle x\rangle & =\int_{-\infty}^{\infty} d \omega e^{i \omega t} \frac{q \tilde{E}_{\text {ext }}(\omega)}{m D(\omega)}=\frac{q \alpha}{2 \pi m} \int_{-\infty}^{\infty} d \omega \frac{e^{i \omega t}}{D(\omega)} \simeq \\
& \simeq \frac{q \alpha}{m \omega_{0}} \sin \left(\omega_{0} t\right) e^{-\frac{\Gamma^{\prime} \omega_{0}^{2}}{2} t}
\end{aligned}
$$

sendo que

$$
\Gamma^{\prime}=\Gamma\left[1+\beta\left(\omega_{0}, y\right)\right]
$$

No cálculo da integral em (4.26), considerou-se que os pólos do integrando têm os valores $\pm \omega_{0}-i \Gamma^{\prime} \omega_{0}^{2} / 2$. Assim se fez uma aproximação semelhante com a feita no cálculo da expressão (4.22).

A nova constante de amortecimento $\Gamma^{\prime}$ é diferente da constante de amortecimento do oscilador no espaço livre $\Gamma$. Portanto, na presença do solenóide, 
os tempos de vida dos estados excitados do oscilador são diferentes do caso no espaço livre. Além disso $\Gamma^{\prime}$ depende da distância $y$ e de sua orientação do verificar que a nova cono uma anisotropia da emissão espontânea. Pode-se uma diminuição dos temte $\Gamma^{\prime}$ é em geral maior do que $\Gamma$, o que leva a aumento da emissão espontân vida dos estados excitados, provocando um

Segundo Blanco, Dechounea.

oscilador através dechoum, França e Santos [1,3], além da excitação do aplicação de uma tõ campo elétrico externo, é possível excitá-lo com a perturbações de tensão conhecida no circuito. Isso é possível, porque as através do campo elétricovocadas no circuito propagam-se até o oscilador uma tensão determinística aplicada pelo solenóide. Assim vamos considerar Então a transformada circuito da forma $\varepsilon_{\text {ext }}(t)=A_{0} \delta(t)$. deve ter o acréscimo do termo

$\tilde{x}_{e x t}(\omega)=-\frac{q}{m} \frac{i A_{0} \omega b(y)}{2 \pi c^{2} Z(\omega) D(\omega)} \simeq-\frac{q A_{0} b(y)}{2 \pi m c^{2} L} \frac{\omega^{2}}{\left(\Omega^{2}-\omega^{2}-i \omega \frac{L}{R}\right)\left(\omega_{0}^{2}-\omega^{2}-i \omega \Gamma^{\prime} \omega_{0}^{2}\right)}$

devido à perturbação determinística da tensão $\varepsilon_{\text {ext }}(t)$. Como $\Gamma \omega_{0} \ll 1 \mathrm{e}$ $R / L \ll \Omega$, a função (4.28) tem os seguintes pólos, $\pm \omega_{0}-i \Gamma^{\prime} \omega_{0}^{2} / 2$ e $\pm \Omega-$ $i R / 2 L$.

A média da posição do oscilador após a excitação da tensão externa do circuito é

$$
\langle x\rangle \simeq \frac{q A_{0} b(y)}{m c^{2} L \rho_{+} \rho_{-}}\left[\omega_{0} \sin \left(\omega_{0} t\right) e^{-\frac{\Gamma^{\prime} \omega_{0}^{2}}{2} t}-\Omega \sin (\Omega t) e^{-\frac{R}{2 L} t}\right],
$$

sendo que

$$
\rho_{ \pm} \equiv \sqrt{\left(\Omega \pm \omega_{0}\right)^{2}+\left(\frac{R}{2 L}-\frac{\Gamma^{\prime} \omega_{0}^{2}}{2}\right)^{2}}
$$

A expressão (4.29) apresenta o comportamento do oscilador após a excitação devida à tensão externa, mostrando o decaimento dos estados excitados de acordo com as constantes $\Gamma^{\prime} \omega_{0}^{2} / 2$ e $R / 2 L$.

Esses resultados, além de serem novas previsões que podem ser verificadas experimentalmente, trazem a possibilidade de se controlar a emissão espontânea de átomos através de mudanças de parâmetros do sistema. 


\subsection{Densidade espectral das tensões flutuan- tes no circuito}

A outra incógnita do sistema de equações, $\tilde{I}_{\text {tot }}(\omega)$, fornece-nos também, pela equação (4.4), a função de correlação da tensão elétrica total do circuito,

$$
\begin{aligned}
& \left\langle\tilde{\varepsilon}_{t o t}(\omega) \tilde{\varepsilon}_{t o t}\left(\omega^{\prime}\right)\right\rangle= \\
& =\frac{\hbar \omega}{2 \pi} \operatorname{coth}\left(\frac{\hbar \omega}{2 K T}\right)\left\{R(\omega)+\frac{3 \Gamma^{2} \omega^{6} b(y)[1+\beta(\omega, y)] .}{2 c\left\{\left(\omega_{0}^{2}-\omega^{2}\right)^{2}+\Gamma^{2} \omega^{6}[1+\beta(\omega, y)]^{2}\right\}}\right\} \delta\left(\omega+\omega^{\prime}\right),
\end{aligned}
$$

sendo que foi usado $\omega_{0}^{\prime} \simeq \omega_{0}$. Nota-se que esta função de correlação é diferente da obtida para o ruído de Nyquist, devido ao segundo termo entre as chaves, causado pelas flutuações do campo magnético gerado pelo oscilador $[1,6]$. Esta contribuição é muito pequena para quase todo o espectro, exceto quando $\omega \approx \omega_{0}$, em que temos um pico muito estreito e elevado. A possibilidade de observação experimental desse pico deve ser levada em consideração, visto que o espectro do ruído de Nyquist já foi medido com bastante precisão [30].

Como $\Gamma \omega_{0}$ é muito pequeno, então é possível fazer a seguinte aproximação

$$
\frac{\omega^{4}[1+\beta(\omega, y)]}{\left(\omega_{0}^{2}-\omega^{2}\right)^{2}+\Gamma^{2} \omega^{6}[1+\beta(\omega, y)]^{2}} \simeq \frac{\pi}{2 \Gamma} \delta\left(\omega-\omega_{0}\right)
$$

Logo a expressão (4.31) torna-se

$$
\left\langle\tilde{\varepsilon}_{t o t}(\omega) \tilde{\varepsilon}_{t o t}\left(\omega^{\prime}\right)\right\rangle \simeq \frac{\hbar \omega}{2 \pi} \operatorname{coth}\left(\frac{\hbar \omega}{2 K T}\right)\left[R(\omega)+\frac{3 \pi c \Gamma \omega_{0}^{2} a^{2} L}{4 l y^{2}} \delta\left(\omega-\omega_{0}\right)\right] .
$$

Espera-se que a contribuição do espectro da tensão originada na interação com oscilador, o segundo termo em (4.33), seja muito pequena para ser medida, devido principalmente ao fator $\Gamma \omega_{0}$, que é muito pequeno. No entanto se considerarmos que o solenóide está imerso em um banho de moléculas dipolares, então, com um cálculo direto $[1,3]$, é possível mostrar que o segundo termo da expressão (4.33) é multiplicado por.

$$
\frac{2 \pi}{3} n a^{2} l \ln \left(\frac{l}{a}\right)
$$

sendo que $n$ é o número de moléculas por unidade de volume. Como $n$ pode ser um número muito grande, então o pico do espectro pode ser mensurável experimentalmente. 


\section{Capítulo 5}

\section{Ruído Radiativo}

Os resultados usuais obtidos para o fenômeno do ruído de Nyquist pressupõe fundamentalmente que o circuito elétrico comporta-se de maneira similar a um oscilador harmônico em equilíbrio termodinâmico com o meio. Em complemento a esta hipótese, pode-se obter o ruído de Nyquist no caso clássico, isto é, no caso de altas temperaturas, aplicando o princípio da equipartição; ou no caso geral, que abrange o limite quântico, usar os resultados da Mecânica Quântica ou da Eletrodinâmica Estocástica para osciladores harmônicos. Como foi apresentado anteriormente, uma das maneiras de estudar teoricamente o ruído de Nyquist é escrevendo a equação de Langevin do circuito elétrico, porém tal procedimento tem a limitação de valer para um circuito muito pequeno, o que pode ser muito diferente de um circuito real.

Recentemente foi feito um artigo por Blanco, França, Santos e Sponchiado [4] procurando entender quais mudanças podem ocorrer no circuito quando consideramos sua estrutura espacial. Nesse trabalho estuda-se o ruído presente em circuitos com indutância, cujo indutor tem uma forma física específica e, se o limite de comprimento de onda longo é usado, ele pode ser considerado macroscópico. Posteriormente foi feito um artigo original por este autor, França e Sponchiado [5], no qual é demonstrado rigorosamente a presença do ruído radiativo em circuitos elétricos com indutância. Nessse trabalho [5], que faz parte desta dissertação, estuda-se a troca de energia do solenóide com os campos eletromagnéticos livre no vácuo. 


\subsection{Ruído radiativo em circuitos com indutância}

O trabalho de Blanco, França, Santos e Sponchiado [4] mostra que para um circuito com um solenóide retilíneo, além do ruído de Nyquist, existe do ruído de Nyquist, deve-se considerar que o circuito está em equilíbrio termodinâmico com a radiação na qual está imerso. Os comprimentos de onda relevantes da radiação eletromagnética, $\lambda$, devem ser muito maiores que um comprimento típico do circuito $l$, isto é, estamos usando a aproximação de comprimentos de onda longos, em acordo com uma análise não-relativística. Todo o estudo realizado é feito no âmbito da Eletrodinâmica Estocástica, pois assim podemos tratar o solenóide, que possue dimensões macróscópicas, de maneira clássica e incluir os aspectos quânticos da radiação, sem cair em inconsistências.

Vamos considerar um solenóide cilíndrico de raio $a$ e comprimento $l$, feito de $N$ espiras com largura desprezível, orientado na direção $z$ de um sistema de coordenadas. Esse solenóide está ligado a um circuito série sem baterias, cuja resistência ôhmica é $R_{o h m}$ e indutância é $L$. Inicialmente vamos desprezar a capacitância do circuito. Se uma densidade de corrente $\vec{J}$ flui através desse solenóide, então surge um momento de dipolo magnético $\vec{\mu}$, tal que

$$
\vec{\mu}=\frac{1}{2 c} \int \vec{r} \times \vec{J} d^{3} r=\frac{\pi N a^{2} I(t)}{c} \hat{\mu}
$$

sendo que $I(t)$ é a corrente elétrica no circuito e $\hat{\mu}=\frac{\vec{\mu}}{\mid \overrightarrow{\mid \overrightarrow{\mid}} \text {. }}$.

A corrente $I(t)$ é um variável estocástica estacionária, assim tomando uma amostra sua em um intervalo de tempo, tal que $0 \leq t \leq T$, então a função $I(t)$ pode ser expandida em uma série de Fourier, ou seja,

$$
I(t)=\sum_{n=-\infty}^{\infty} I_{n} e^{i \omega_{n} t}
$$

dadas as freqüências $\omega_{n}=\frac{2 \pi n}{T}$, com $n \in\{\ldots,-2,-1,0,1,2, \ldots\}$. Os coeficientes de Fourier estão representados por $I_{n}$.

Um sistema físico com um dipolo magnético emite radiação, levando a uma dissipação de energia. A potência correspondente pode ser calculada [16]. Para o caso da corrente elétrica do solenóide ser dada pela expressão (5.2), a potência emitida pode ser escrita como uma série de Fourier, cujos 
coeficientes são

$$
P_{n}=\frac{\omega_{n}^{4}}{3 c^{3}}|\vec{\mu}|^{2}=\frac{\pi^{2} N^{2}}{3 c}\left(\frac{a \omega_{n}}{c}\right)^{4} I_{n}^{2}=\frac{R_{n} I_{n}^{2}}{2}
$$

sendo que é definida uma resistência radiativa $R_{n}$ para cada corrente $I_{n}$.

No limite do contínuo, a série de Fourier de $I(t)$, (5.2), torna-se uma transformada de Fourier, e o intervalo de tempo da expansão tende a ser do se, no caso contínuo,

$$
P_{\omega}=\frac{\pi^{2} N^{2}}{3 c}\left(\frac{a \omega}{c}\right)^{4} \tilde{I}_{\omega}^{2}=\frac{R(\omega) \tilde{I}_{\omega}^{2}}{2}
$$

Então, a partir da expressão (5.4), a resistência radiativa de um solenóide com as características consideradas é

$$
R_{\text {rad }}(\omega)=\frac{2 \pi^{2} N^{2}}{3 c}\left(\frac{a \omega}{c}\right)^{4}
$$

Logo a resistência total do circuito é dada por

$$
R(\omega, T)=R_{\text {ohm }}(\omega, T)+R_{\text {rad }}(\omega)
$$

considerando que a resistência ôhmica $R_{\text {ohm }}(\omega, T)$ depende tanto da temperatura como da freqüência de oscilação do circuito. Deve-se notar que a resistência ôhmica é a dissipação correspondente às flutuações elétricas medidas por Johnson [19] e explicadas por Nyquist [20]. Analogamente devem existir flutuações elétricas correspondentes à resistência radiativa.

As flutuações da corrente e da tensão associadas à resistência radiativa têm sua origem na interação entre o solenóide e os campos eletromagnéticos livres no vácuo. Isto mostra-se usando a função de correlação do campo magnético livre, que, como estamos no âmbito da SED, é a expressão (2.36) apresentada no capítulo 1. Pela aproximação do limite de comprimentos de onda longos, que estamos usando, a expressão (2.36) pode ser reduzida para o caso de campos independentes da posição, ou seja,

$$
\left\langle B_{i}\left(t_{1}\right) B_{j}\left(t_{2}\right)\right\rangle_{T}=\int_{-\infty}^{\infty} d \omega \delta_{i j} \frac{\hbar \omega^{3}}{3 \pi c^{3}} \operatorname{coth}\left(\frac{\hbar \omega}{2 K T}\right) e^{i \omega\left(t_{1}-t_{2}\right)}
$$

O campo magnético é considerado como tendo um ruído gaussiano e média igual à zero. Assim, usando as relações de Wiener-Khintchine, a função de 
correlação da transformada de Fourier da componente no eixo $0-z$ do campo
magnético é

$$
\left\langle\tilde{B}_{z}(\omega) \tilde{B}_{z}\left(\omega^{\prime}\right)\right\rangle=S_{F V_{B}}(\omega) \delta\left(\omega+\omega^{\prime}\right)=\frac{\hbar \omega^{3}}{3 \pi c^{3}} \operatorname{coth}\left(\frac{\hbar \omega}{2 K T}\right) \delta\left(\omega+\omega^{\prime}\right) .
$$

O campo magnético livre, por seu caráter aleatório, provoca uma indução de força eletromotriz aleatória ao longo das espiras circulares do solenóide,
expresso por

$$
\varepsilon_{B}(t)=-\frac{\pi a^{2} N}{c} \frac{\partial B_{z}(t)}{\partial t}
$$

Então, combinando as expressões (5.8) e (5.9), é possível escrever que a densidade espectral da tensão elétrica flutuante causado pelo campo magnético livre é

$$
\begin{aligned}
S_{B}(\omega, T) & =\left(\frac{\pi a^{2} N}{c}\right)^{2} \omega^{2} S_{F V_{B}}(\omega, T) \\
& =\frac{2 \pi N^{2}}{3 c}\left(\frac{a \omega}{c}\right)^{4} \frac{\hbar \omega}{2} \operatorname{coth}\left(\frac{\hbar \omega}{2 K T}\right) .
\end{aligned}
$$

Para simplificação de notação, neste capítulo, as densidades espectrais de tensão possuem apenas índices que indicam sua origem. É usado o índice $B$ para as flutuações geradas pela indução do vácuo sobre o solenóide e o índice $N$ para o ruído de Nyquist.

É evidente que a expressão (5.10) pode ser arranjada, de modo que a resistência radiativa, dada por (5.5), pode ser explicitada, fornecendo então a sua correspondente relação flutuação-dissipação,

$$
S_{B}(\omega, T)=\frac{\hbar \omega R_{\text {rad }}(\omega)}{2 \pi} \operatorname{coth}\left(\frac{\hbar \omega}{2 K T}\right)
$$

Note que, fisicamente, ao mesmo tempo que o campo magnético provoca flutuações no circuito, ocorre a dissipação do sistema devido ao dipolo magnético flutuante. No estado estacionário, o balanço entre a dissipação e a flutuação do circuito obedece a expressão (5.11).

Após este passo, há condições de encontrar o teorema flutuação-dissipação para a tensão elétrica total do circuito, que deve ser

$$
\varepsilon(t)=\varepsilon_{N}(t)+\varepsilon_{B}(t)
$$


As flutuações de origens diferentes são supostas não correlacionadas, isto é, $\left\langle\varepsilon_{N}(t) \varepsilon_{B}(t)\right\rangle=0$. Isso é justificável, porque o ruído de Nyquist surge deve surgir da inducão dós fios do circuito, e o novo ruído considerado especificamente do direta da radiação térmica e de ponto-zero, mais $\langle\varepsilon(t)\rangle=0$, a distribuição magnético do vácuo, no solenóide. Portanto, como

$$
\begin{aligned}
S_{\varepsilon}(\omega, T) & =S_{N}(\omega, T)+S_{B}(\omega, T)= \\
& =\frac{\hbar \omega}{2 \pi}\left[R_{o h m}(\omega, T)+\frac{2 \pi^{2} N^{2}}{3 c}\left(\frac{a \omega}{c}\right)^{4}\right] \operatorname{coth}\left(\frac{\hbar \omega}{2 K T}\right) .
\end{aligned}
$$

Então a relação flutuação-dissipação para a dissipação total do circuito é

$$
S_{\varepsilon}(\omega, T)=\frac{\hbar \omega R(\omega, T)}{2 \pi} \operatorname{coth}\left(\frac{\hbar \omega}{2 K T}\right) .
$$

Com estes resultados é possivel verificar o comportamento de um circuito RLC série sem baterias, cujo solenóide tem dimensões macroscópicas. Isto foi feito para se estudar os efeitos deste novo ruído em um circuito com propriedades realísticas de resistência, indutância e capacitância. Além disto, para a prova do ruído de Nyquist, Ginzburg [23] utilizou a condição de equilíbrio e a energia média de um circuito RLC, no limite de resistência pequena, na qual o circuito se comporta como um oscilador harmônico.

Seguindo as considerações anteriores, o circuito estudado tem dimensões muito pequenas comparadas aos comprimentos de onda correspondentes à faixa de freqüências em questão. Nestas condições os campos e correntes do circuito estão na aproximação quase-estacionária, na qual a corrente elétrica $I(t)$ é a mesma em todo o circuito, num dado instante $t$. Todo o cálculo a seguir foi feito como em [23].

A energia média no circuito é dada pela expressão bem conhecida

$$
\begin{aligned}
\langle W\rangle & =L\left\langle I^{2}(t)\right\rangle=L \int_{-\infty}^{+\infty} S_{I}(\omega, T) d \omega= \\
& =2 L \int_{0}^{+\infty} \frac{C^{2} \omega^{2} S_{\varepsilon}(\omega, T)}{C^{2} \omega^{2} R^{2}(\omega, T)+\left(L C \omega^{2}-1\right)^{2}} d \omega .
\end{aligned}
$$

Deve-se considerar que a resistência total deve ser pequena $R(\omega, T) \rightarrow 0$. Desse modo podemos escrever a condição

$$
\frac{R(\omega, T)}{L \Omega}=C \Omega R(\omega, T)<<1
$$


sendo que $\Omega=(L C)^{-1 / 2}$ é a freqüência característica do circuito. Assim a
equação (5.10) é, aproximadamente,

$$
\langle U\rangle \simeq \pi \frac{S_{\varepsilon}(\omega, T)}{R(\omega, T)}=\frac{\hbar \Omega}{2} \operatorname{coth}\left(\frac{\hbar \Omega}{2 K T}\right)
$$

Logo o circuito com a resistência radiativa tem a mesma energia média que um oscilador harmônico em equilíbrio com radiação térmica e de ponto-zero. Isso foi uma das condições necessárias para que na referência [23] se obtivesse a densidade espectral do ruído de Nyquist.

Pode-se ver na expressão (5.13), que o ruído devido à resistência radiativa contribui com a quarta potência da freqüência. Isto nos indica que, apesar desta contribuição ser muito pequena para baixas freqüências, ela se torna muito relevante para freqüências altas, como podemos ver nos gráficos da referência [4]. Este resultado abre a possibilidade do ruído da resistência radiativa ser verificado experimentalmente.

Além do ruído radiativo em circuito com indutância, foi mostrado recentemente por França e Santos [7], que átomos com dipolos magnéticos podem trocar energia e momento angular e linear com o campo do vácuo e o solenóide. Foi mostrado também [8] que em algumas situações as energias nos átomos magnéticos dipolares e no solenóide crescem simultaneamente. Neste caso os campos do vácuo provêem energia para ambos os sistemas. Todos estes efeitos poderiam ser verificados experimentalmente.

\subsection{Demostração da presença do ruído radi- ativo}

\subsubsection{Introdução}

Para esclarecer a relação entre as flutuações de corrente microscópicas e o conceito macroscópico de resistência radiativa, durante a elaboração desta dissertação, foi feito um estudo que resultou na publicação de um trabalho original, em colaboração com França e Sponchiado [5]. Nesse trabalho, apresentado nesta seção, as flutuações do circuito correspondentes à resistência radiativa, preditas por Blanco, França, Santos e Sponchiado [4], são analisadas a partir da troca de energia do circuito com os campos eletromagnéticos livre no vácuo. 
O método usado para estudar a interação do circuito RLC série com a radiação térmica e de ponto-zero consiste em calcular o vetor de Poynting em todo o espaço em torno do circuito. É mostrado a seguir que a presença do circuito constituído de um solenóide macroscópico cilíndrico produz uma o circuito deve estar da radiação do vácuo. Porém, no regime estacionário, desse modo não há flux equilíbrio termodinâmico com o banho de radiação, vácuo a sua volta. Isso signifio de energia entre o circuito e a radiação do do campo eletromagnético resultae a média temporal do vetor de Poynting

$$
\langle\vec{S}\rangle=0
$$

em qualquer ponto do espaço. Consequentemente, como é esperado fisicamente, o espectro da radiação do vácuo não se altera ao longo do tempo e nenhuma radiação pode ser detectada surgindo do circuito. Além disso, com a condição (5.18), os ruídos no circuito e suas correspondentes resistências ôhmica e radiativa são consistentemente necessárias.

Vamos considerar todas as aproximações e os arranjos da seção anterior. O circuito possue uma resistência ôhmica, que depende da temperatura e da freqüência de oscilação da corrente, porém estes aspectos não são relevantes nesta análise. Apenas devemos lembrar de que a resistência ôhmica está relacionada com o ruído de Nyquist, assim vamos escrever

$$
R_{\text {ohm }}=R_{N} \text {. }
$$

A impedância total do circuito pode ser escrita como

$$
Z(\omega)=R(\omega)+i\left(\omega L-\frac{1}{\omega C}\right)
$$

sendo que a resistência total do circuito $R(\omega)$ é ainda desconhecida. É possível dizer apenas que a resistência pode depender da freqüência e possue uma parte ôhmica.

\subsubsection{Cálculo dos campos elétrico e magnético gerados pelo solenóide}

As flutuações eletromagnéticas do vácuo produzem flutuações na tensão do circuito através da ação do campo magnético livre nas espiras do solenóide. 
Assim esta nova fonte de ruído produz uma tensão elétrica $\varepsilon_{B}(t)$ que é acrescentada à tensão do ruído de Nyquist $\varepsilon_{N}(t)$, valendo a expressão (5.12). Logo as transformadas o mesmo para as respectivas correntes $I_{B}(t)$ e $I_{N}(t)$. o fourier destas variáveis relacionam-se por

$$
\tilde{I}(\omega)=\tilde{I}_{N}(\omega)+\tilde{I}_{B}(\omega)=\frac{1}{Z(\omega)}\left[\tilde{\varepsilon}_{N}(\omega)+\tilde{\varepsilon}_{B}(\omega)\right]
$$
Pelos motivos já citados na seção anterior, os ruídos de origem diferentes não
são correlacionados, isto é,

$$
\begin{aligned}
& \left\langle\tilde{\varepsilon}_{N}(\omega) \tilde{\varepsilon}_{B}(\omega)\right\rangle=0, \\
& \left\langle\tilde{I}_{N}(\omega) \tilde{I}_{B}(\omega)\right\rangle=0 .
\end{aligned}
$$

A contribuição do ruído de Nyquist para a corrente no circuito segue a seguinte função de correlação, que pode ser obtida das expressões (3.16) e (3.32),

$$
\left\langle I_{N}(t) I_{N}\left(t^{\prime}\right)\right\rangle=\int_{-\infty}^{+\infty} d \omega \frac{R_{N}}{|Z(\omega)|^{2}} \frac{\hbar \omega}{2 \pi} \operatorname{coth}\left(\frac{\hbar \omega}{2 K T}\right) e^{i \omega\left(t-t^{\prime}\right)}
$$

Um circuito com um solenóide com a geometria considerada deve ter um momento de dipolo magnético determinado por (vide [16])

$$
\vec{\mu}=\frac{1}{2 c} \int \vec{r} \times \vec{J} d^{3} r=\frac{\pi N a^{2}}{c}\left[I_{N}(t)+I_{B}(t)\right] \hat{\mu},
$$

visto que $\vec{J}$ é a densidade de corrente no circuito. Devemos notar que a corrente total do circuito pode ser dividida na parte com origem no ruído de Nyquist e na parte com origem na indução dos campos livres no vácuo, de modo que os campos gerados pelo dipolo magnético do solenóide também podem ser calculados separando as contribuições de origens diferentes.

O campo elétrico gerado pela parte do dipolo magnético do solenóide devido ao ruído de Nyquist é (veja $[13,16]$ )

$$
\vec{E}_{N}(\vec{r}, t)=-2 \int_{0}^{\infty} d \omega \frac{\pi N a^{2}}{c} \tilde{I}_{N}(\omega) e^{-i \omega t} \vec{H}
$$

sendo que são definidos os vetores

$$
\vec{H} \equiv k^{3} e^{i k r} \hat{n} \times \hat{\mu}\left[\frac{1}{k r}+\frac{i}{(k r)^{2}}\right]
$$


e $\hat{n} \equiv \frac{\vec{r}}{r}$. O correspondente campo magnético é

$$
\vec{B}_{N}(\vec{r}, t)=2 \int_{0}^{\infty} d \omega \frac{\pi N a^{2}}{c} \tilde{I}_{N}(\omega) e^{-i \omega t} \vec{G}
$$

sendo que

$$
\vec{G} \equiv k^{3} e^{i k r}\left\{(\hat{n} \times \hat{\mu}) \times \hat{n}\left(\frac{1}{k r}\right)+[3 \hat{n}(\hat{n} \cdot \hat{\mu})-\hat{\mu}]\left[\frac{1}{(k r)^{3}}-\frac{i}{(k r)^{2}}\right]\right\}
$$

gerados pelo dipolo magnético com origem na indução dos campos livres no vácuo sobre o solenóide, é necessário obter a correspondente corrente elétrica $I_{B}$. Para isso o campo elétrico livre pode ser escrito como

$$
\vec{E}_{V F}(\vec{r}, t)=-\Re e \sum_{\alpha=1}^{2} \int d^{3} k \mathfrak{h}(\vec{k}, T) e^{-i \omega t+i \vec{k} \cdot \vec{r}+i \theta(\vec{k}, \alpha)} \frac{\vec{k} \times \hat{\epsilon}(\vec{k}, \alpha)}{k},
$$

e o campo magnético livre pode ser expresso como

$$
\vec{B}_{V F}(\vec{r}, t)=\Re e \sum_{\alpha=1}^{2} \int d^{3} k \mathfrak{h}(\vec{k}, T) e^{-i \omega t+i \vec{k} \cdot \vec{r}+i \theta(\vec{k}, \alpha)} \hat{\epsilon}(\vec{k}, \alpha) .
$$

As expressões (5.29) e (5.30) diferem apenas na orientação dos versores de polarização das expressões (2.1) e (2.2).

Combinando-se as equações (3.14) e (5.9), a corrente induzida no solenóide pelo campo magnético (5.30) é (veja [16])

$$
I_{B}(t)=\Re e \sum_{\alpha=1}^{2} \int d^{3} k \frac{i \omega \pi N a^{2}}{c Z(\omega)} \mathfrak{h} e^{-i \omega t+i 0}(\hat{\epsilon} \cdot \hat{\mu}) .
$$

Assim, os campos devidos a corrente induzida pelos campos livres do vácuo, $I_{B}(t)$, podem ser calculados. O campo elétrico é

$$
\vec{E}_{B}(\vec{r}, t)=-\Re e \sum_{\alpha=1}^{2} \int d^{3} k i \omega\left(\frac{\pi N a^{2}}{c}\right)^{2} \frac{\mathfrak{h}}{Z(\omega)} e^{-i \omega t+i \theta}(\hat{\epsilon} \cdot \hat{\mu}) \vec{H}
$$

e o respectivo campo magnético é

$$
\vec{B}_{B}(\vec{r}, t)=\Re e \sum_{\alpha=1}^{2} \int d^{3} k i \omega\left(\frac{\pi N a^{2}}{c}\right)^{2} \frac{\mathfrak{h}}{Z(\omega)} e^{-i \omega t+i \theta}(\hat{\epsilon} \cdot \hat{\mu}) \vec{G}
$$

55 
Logo os campos elétrico e magnético resultantes em qualquer ponto do
espaço são

$$
\begin{aligned}
& \vec{E}(\vec{r}, t)=\vec{E}_{F V}(\vec{r}, t)+\vec{E}_{N}(\vec{r}, t)+\vec{E}_{B}(\vec{r}, t), \\
& \vec{B}(\vec{r}, t)=\vec{B}_{F V}(\vec{r}, t)+\vec{B}_{N}(\vec{r}, t)+\vec{B}_{B}(\vec{r}, t) .
\end{aligned}
$$

A partir da expressão (5.34), podemos verificar se a distribuição expectral do campo eletromagnético resultante próximo do solenóide é diferente do caso de campos eletromagnéticos livres. Para isso vamos calcular a função de correlação do campo elétrico resultante, considerando que o eixo do solenóide é paralelo à direção $0-z$. Assim, para as componentes $i$ e $\alpha$ (nas direções $x$, $y$ ou $z$ ) do campo elétrico $\vec{E}(\vec{r}, t)$, temos

$$
\begin{array}{r}
\left\langle E_{i}\left(\vec{r}_{1}, t_{1}\right) E_{\alpha}\left(\vec{r}_{2}, t_{2}\right)\right\rangle=\int d^{3} k\left(\delta_{i \alpha}-\frac{k_{i} k_{\alpha}}{k^{2}}\right) \frac{\hbar \omega}{4 \pi^{2}} \operatorname{coth}\left(\frac{\hbar \omega}{2 K T}\right) \times \\
\times \cos \left[\omega\left(t_{1}-t_{2}\right)-\vec{k} \cdot\left(\vec{r}_{1}-\vec{r}_{2}\right)\right]+ \\
+\frac{4}{\pi} \int_{0}^{\infty} d \omega\left[R_{N}+\frac{2 \pi^{2} N^{2}}{3 c}\left(\frac{a \omega}{c}\right)^{4}\right] n_{j} n_{\beta} \epsilon_{i j z} \epsilon_{\alpha \beta z}\left(\frac{\pi N a^{2}}{c}\right)^{2} \times \\
\times\left(\frac{1}{r^{2}}+\frac{c^{2}}{\omega^{2} r^{4}}\right) \frac{\hbar \omega^{5}}{c^{4}|Z(\omega)|^{2}} \operatorname{coth}\left(\frac{\hbar \omega}{2 K T}\right) \times \\
\times \cos \left[\omega\left(t_{1}-t_{2}\right)-\frac{\omega}{c}\left(r_{1}-r_{2}\right)\right] .
\end{array}
$$

Foi desprezada a contribuição dos termos cruzados entre $\vec{E}_{F V}$ e $\vec{E}_{B}$, pois, no limite de comprimentos de onda longos, esses campos são correlacionados apenas sobre o solenóide. A função de correlação obtida para o campo elétrico resultante devido a presença do solenóide é claramente diferente da função de correlação do campo elétrico livre. Nota-se também que o campo elétrico resultante é anisotrópico, por causa das contribuições dos campos de dipolo magnético gerados pelo solenóide. Com a simples aplicação das relações de Wiener-Khintchine, verifica-se que o espectro do campo elétrico é diferente do caso no espaço livre.

\subsubsection{A média do vetor de Poynting}

Determinados os campos elétrico e magnético resultantes, podemos, então, calcular o vetor de Poynting $\vec{S}$ e o seu valor médio. O vetor de Poynting é dado por

$$
\vec{S}(\vec{r}, t)=\frac{c}{4 \pi}(\vec{E}(\vec{r}, t) \times \vec{B}(\vec{r}, t)) .
$$


Devido à natureza dos campos elétrico e magnético envolvidos, o vetor de de Poynting que nos interessa*é variável aleatória. Assim a média do vetor campos, o que pode ser obtido de mobre o tempo e as fases aleatórias dos e magnético na forma complexa. estacionário, então não há perda de imo o sistema estudado está no regime médio. A quantidade procurada é forformação relevante no cálculo do valor

$$
\begin{aligned}
\langle\vec{S}\rangle & =\frac{c}{8 \pi} \Re e\langle\vec{E} \times \vec{B}\rangle= \\
& =\frac{c}{8 \pi} \Re e\left[\left\langle\vec{E}_{V F}^{*} \times \vec{B}_{V F}\right\rangle+\left\langle\vec{E}_{B}^{*} \times \vec{B}_{B}\right\rangle+\right. \\
& \left.+\left\langle\vec{E}_{V F}^{*} \times \vec{B}_{B}\right\rangle+\left\langle\vec{E}_{B}^{*} \times \vec{B}_{V F}\right\rangle+\left\langle\vec{E}_{N}^{*} \times \vec{B}_{N}\right\rangle\right] .
\end{aligned}
$$

Os outros termos da média do vetor de Poynting,

$$
\begin{array}{ccc}
\left\langle\vec{E}_{F V}^{*} \times \vec{B}_{N}\right\rangle=0, & \left\langle\vec{E}_{B}^{*} \times \vec{B}_{N}\right\rangle=0, \\
\left\langle\vec{E}_{N}^{*} \times \vec{B}_{F V}\right\rangle=0 & \text { e } & \left\langle\vec{E}_{N}^{*} \times \vec{B}_{B}\right\rangle=0
\end{array}
$$

são nulos, pois os campos gerados pelo ruído de Nyquist não são correlacionados com os campos gerados pela radiação do vácuo.

É possível mostrar que os termos da equação (5.37), sem incluir o termo devido ao ruído de Nyquist, tornam-se (veja [13])

$$
\begin{gathered}
\left\langle\vec{E}_{V F}^{*} \times \vec{B}_{V F}\right\rangle=\int d^{3} k \mathfrak{h}^{2}\left[\sum_{\alpha=1}^{2} \hat{\epsilon} \times\left(\frac{\vec{k} \times \hat{\epsilon}}{k}\right)\right] \\
\left\langle\vec{E}_{B}^{*} \times \vec{B}_{B}\right\rangle=-\int d^{3} k\left(\frac{\pi N a^{2}}{c}\right)^{4} \frac{\omega^{2} \mathfrak{h}^{2}}{|Z(\omega)|^{2}} \vec{H}^{*} \times \vec{G}\left[\sum_{\alpha=1}^{2}(\hat{\epsilon} \cdot \hat{\mu})^{2}\right] \\
\left\langle\vec{E}_{V F}^{*} \times \vec{B}_{B}\right\rangle=-\int d^{3} k\left(\frac{\pi N a^{2}}{c}\right)^{2} \frac{i \omega \mathfrak{h}^{2}}{|Z(\omega)|^{2}}\left(-Z^{*}(\omega) \vec{G}\right) \times\left[\sum_{\alpha=1}^{2} \frac{\vec{k} \times \hat{\epsilon}}{k}(\hat{\epsilon} \cdot \hat{\mu}) e^{-i \vec{k} \cdot \vec{r}}\right]
\end{gathered}
$$

e

$$
\left\langle\vec{E}_{B}^{*} \times \vec{B}_{V F}\right\rangle=-\int d^{3} k\left(\frac{\pi N a^{2}}{c}\right)^{2} \frac{i \omega \mathfrak{h}^{2}}{|Z(\omega)|^{2}}\left(Z(\omega) \vec{H}^{*}\right) \times\left[\sum_{\alpha=1}^{2} \hat{\epsilon}(\hat{\epsilon} \cdot \hat{\mu}) e^{i \vec{k} \cdot \vec{r}}\right]
$$


Como a soma sobre as polarizações deve seguir as relações entre os verangulares das expacão e o vetor de onda dadas em (2.3), então as integrais (5.39) a (5.42) são, respectivamente,

$$
\begin{gathered}
\int d \Omega_{\vec{k}}\left[\sum_{\alpha=1}^{2} \hat{\epsilon} \times\left(\frac{\vec{k} \times \hat{\epsilon}}{k}\right)\right]=\int d \Omega_{\vec{k}} \frac{2 \vec{k}}{k}=0, \\
\int d \Omega_{\vec{k}}\left[\sum_{\alpha=1}^{2}(\hat{\epsilon} \cdot \hat{\mu})^{2}\right]=\int d \Omega_{\vec{k}}\left[1-\left(\frac{\vec{k} \cdot \hat{\mu}}{k}\right)\right]=\frac{8 \pi}{3}, \\
\int d \Omega_{\vec{k}}\left[\sum_{\alpha=1}^{2} \frac{\vec{k} \times \hat{\epsilon}}{k}(\hat{\epsilon} \cdot \hat{\mu}) e^{-i \vec{k} \cdot \vec{r}}\right]=\int d \Omega_{\vec{k}} \frac{\vec{k} \times \hat{\mu}}{k} e^{-i \vec{k} \cdot \vec{r}}=\frac{4 \pi i}{k^{3}} \Re e \vec{H}
\end{gathered}
$$

$$
\int d \Omega_{\vec{k}}\left[\sum_{\alpha=1}^{2} \hat{\epsilon}(\hat{\epsilon} \cdot \hat{\mu}) e^{i \vec{k} \cdot \vec{r}}\right]=\int d \Omega_{\vec{k}}\left[\hat{\mu}-\frac{\vec{k}(\vec{k} \cdot \hat{\mu})}{k^{2}}\right] e^{i \vec{k} \cdot \vec{r}}=\frac{4 \pi}{k^{3}} \mathfrak{I m} \vec{G}
$$

O termo de (5.37) devido ao ruído de Nyquist é

$$
\begin{aligned}
\left\langle\vec{E}_{N}^{*} \times \vec{B}_{N}\right\rangle & =-4 \int_{0}^{\infty} d \omega \int_{0}^{\infty} d \omega^{\prime}\left(\frac{\pi N a^{2}}{c}\right)^{2}\left\langle\tilde{I}_{N}(\omega) \tilde{I}_{N}\left(\omega^{\prime}\right)\right\rangle \vec{H}^{*} \times \vec{G}= \\
& =-4 \int_{0}^{\infty} d \omega\left(\frac{\pi N a^{2}}{c}\right)^{2} \frac{\hbar \omega R_{N}}{2 \pi|Z(\omega)|^{2}} \operatorname{coth}\left(\frac{\hbar \omega}{2 K T}\right) \vec{H}^{*} \times \vec{G}= \\
& =-\int_{0}^{\infty} d \omega\left(\frac{\pi N a^{2}}{c}\right)^{2} \frac{4 \pi R_{N} h^{2}}{|Z(\omega)|^{2}} \vec{H}^{*} \times \vec{G}
\end{aligned}
$$

Para os outros termos de (5.37), as intergais angulares de (5.43) a (5.46) são calculadas escolhendo $\vec{r}$ na direção do eixo $0-z$ e variando a direção do dipolo magnético $\hat{\mu}$, além de se usar coordenadas esféricas. Os resultados obtidos são

$$
\begin{gathered}
\left\langle\vec{E}_{V F}^{*} \times \vec{B}_{V F}\right\rangle=0 \\
\left\langle\vec{E}_{B}^{*} \times \vec{B}_{B}\right\rangle=-\int_{0}^{\infty} d \omega \frac{8 \pi \omega^{4}}{3 c^{3}}\left(\frac{\pi N a^{2}}{c}\right)^{4} \frac{\mathfrak{h}^{2}}{|Z(\omega)|^{2}} \vec{H}^{*} \times \vec{G} \\
\left\langle\vec{E}_{V F}^{*} \times \vec{B}_{B}\right\rangle=-\int_{0}^{\infty} d \omega \frac{i \omega^{3}}{c^{3}}\left(\frac{\pi N a^{2}}{c}\right)^{2} \frac{\mathfrak{h}^{2}}{|Z(\omega)|^{2}}\left(-Z^{*}(\omega) \vec{G}\right) \times\left(\frac{4 \pi i}{k^{3}} \Re \vec{H}\right)
\end{gathered}
$$




$$
\left\langle\vec{E}_{B}^{*} \times \vec{B}_{V F}\right\rangle=-\int_{0}^{\infty} d \omega \frac{i \omega_{0}^{3}}{c^{3}}\left(\frac{\pi N a^{2}}{c}\right)^{2} \frac{h^{2}}{|Z(\omega)|^{2}}\left(Z(\omega) \vec{H}^{*}\right) \times\left(\frac{4 \pi}{k^{3}} \Im \mathrm{m} \vec{G}\right) .
$$

Como

$$
\Re e\left(Z^{*}(\omega) \vec{G}\right)=\operatorname{Re}(Z(\omega)) \operatorname{Re}(\vec{G})+\operatorname{Im}(Z(\omega)) \operatorname{Im}(\vec{G})
$$

e

$$
\Re e\left(i Z(\omega) \vec{H}^{*}\right)=\operatorname{Re}(Z(\omega)) \operatorname{Im}(\vec{H})-\operatorname{Im}(Z(\omega)) \Re e(\vec{H}),
$$

então os resultados obtidos de (5.47) a (5.51) podem ser colecionados e manipulados na expressão (5.37), da qual se obtem

$$
\begin{array}{r}
\langle\vec{S}\rangle=-\frac{c}{2}\left(\frac{\pi N a^{2}}{c}\right)^{2} \int_{0}^{\infty} d \omega \frac{\mathfrak{h}^{2}}{|Z(\omega)|^{2}}\left[\frac{2 \pi^{2} N^{2}}{3 c}\left(\frac{a \omega}{c}\right)^{4}+R_{N}-\Re e Z(\omega)\right] . \\
\cdot(\Re e \vec{H} \times \Re e \vec{G}+\operatorname{Im} \vec{H} \times \operatorname{Im} \vec{G}) .
\end{array}
$$

Esta expressão só é nula quando o integrando é nulo. Isto apenas é possível se

$$
\begin{aligned}
\Re e Z(\omega)=R(\omega) & =\frac{2 \pi^{2} N^{2}}{3 c}\left(\frac{a \omega}{c}\right)^{4}+R_{N} \\
& =R_{\text {rad }}(\omega)+R_{N}
\end{aligned}
$$

Isto significa que mesmo com a presença do circuito, que está absorvendo e emitindo energia da radiação eletromagnética a sua volta, não existe transferência média de energia em qualquer direção, qualquer que seja a freqüência. Logo a distribuição espectral dos campos do vácuo é estável, apesar da presença do solenóide. Aplicando o teorema flutuação-dissipação para a resistência total obtida em (5.55), conclue-se que, além do ruído de Nyquist, o ruído radiativo deve necessariamente existir no particular sistema analisado aqui.

\subsubsection{Condições para a medição do ruído radiativo}

Demonstrada a existência do ruído radiativo em circuitos com indutância e suas conseqüências, devemos procurar saber em quais situações esse ruído 
torna-se relevante. Inicialmente vamos definir um índice de proporção entre as intensidades do ruído total e do ruído de Nyquist e denominá-lo por $\xi(\omega)$.
Usando as distribuições espectrais das tensões, temos

$$
\xi(\omega) \equiv \frac{S_{\varepsilon}}{S_{N}}=1+\frac{2 \pi^{2} N^{2}}{3 c R_{o h m}}\left(\frac{a \omega}{c}\right)^{4},
$$

sendo que $S_{\varepsilon}$ é o espectro em (5.13) e $S_{N}$ é o espectro em (3.3). Quanto maior o valor de $\xi$, maior é a contribuição do ruído radiativo no ruído total

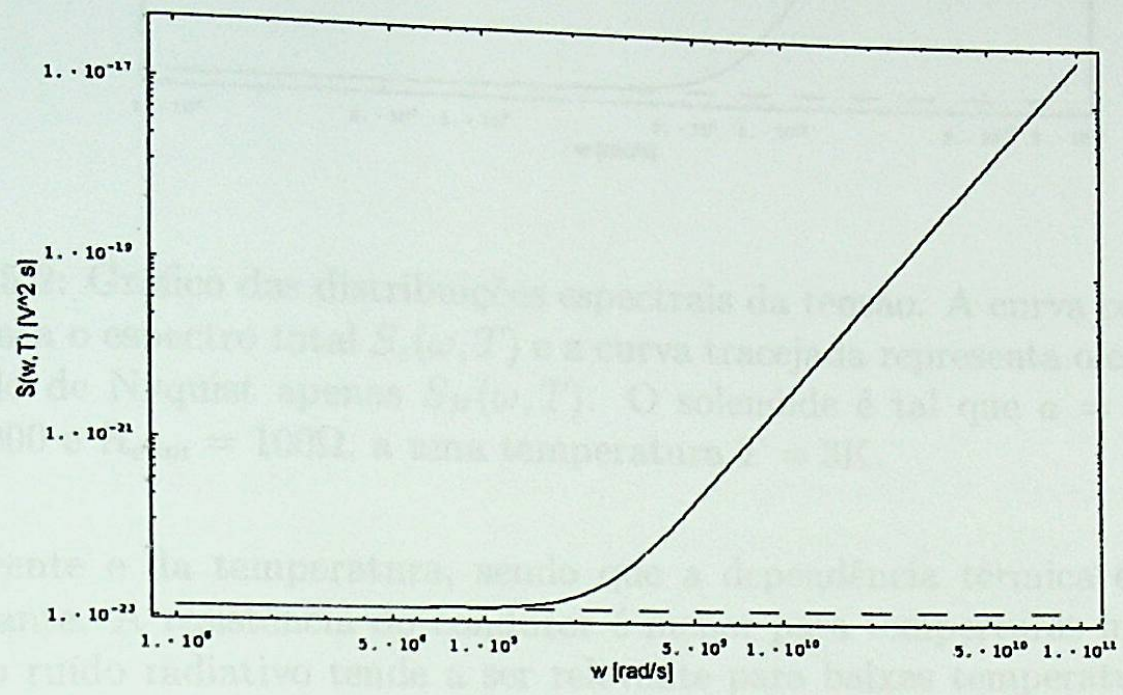

Figura 5.1: Gráfico das distribuições espectrais da tensão. A curva contínua representa o espectro total $S_{\varepsilon}(\omega, T)$ e a curva tracejada representa o espectro do ruído de Nyquist apenas $S_{N}(\omega, T)$. O solenóide é tal que $a=0,1 \mathrm{~cm}$, $N=1000$ e $R_{\text {ohm }}=1 \Omega$, a uma temperatura $T=3 \mathrm{~K}$.

Como se vê em (5.56), para que $\xi$ seja grande, a resistência ôhmica deve ser a menor possível. Uma expressão muito conhecida da resistência ôhmica em um fio condutor é

$$
R_{o h m}(\omega, T)=\frac{d}{\sigma(\omega, T) A}=\frac{2 \pi N a}{\sigma(\omega, T) A},
$$

sendo que $d=2 \pi N a$ é o comprimento total do fio, cuja área de seção reta é $A$. A condutividade do fio, $\sigma(\omega, T)$, depende da freqüência de oscilação 


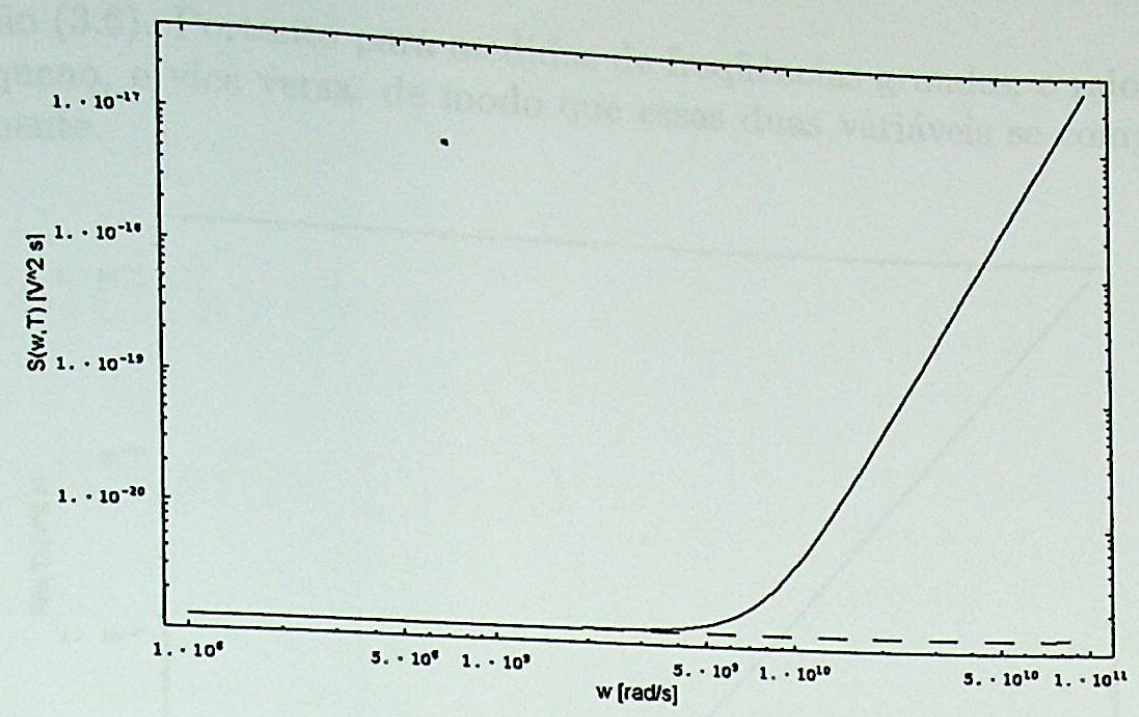

Figura 5.2: Gráfico das distribuições espectrais da tensão. A curva contínua representa o espectro total $S_{\varepsilon}(\omega, T)$ e a curva tracejada representa o espectro do ruído de Nyquist apenas $S_{N}(\omega, T)$. O solenóide é tal que $a=0,1 \mathrm{~cm}$, $N=1000$ e $R_{\text {ohm }}=100 \Omega$, a uma temperatura $T=3 \mathrm{~K}$.

da corrente e da temperatura, sendo que a dependência tërmica é muito importante. A resistência do condutor é menor para temperturas menores, assim o ruído radiativo tende a ser relevante para baixas temperaturas. A figura 5.1 apresenta os gráficos do espectro total $S_{\varepsilon}$ e do espectro de Nyquist $S_{N}$ para uma resistência ôhmica de $1 \Omega$ (as demais características do solenóide são apresentadas na legenda da figura). Podemos notar que $S_{\varepsilon}$ se diferencia consideravelmente de $S_{N}$ a partir aproximadamente da freqüência de $2 \cdot 10^{9} \mathrm{rad} / \mathrm{s}$. Para o mesmo solenóide, porém com uma resistência ôhmica de $100 \Omega$, podemos ver na figura 5.2 que $S_{\varepsilon}$ se destaca do ruído de Nyquist aproximadamente a partir de $5 \cdot 10^{9} \mathrm{rad} / \mathrm{s}$.

A expressão (5.56) revela também que quanto maior o raio do solenóide $a$, maior é a importância do ruído radiativo. O mesmo aspecto notamos para a freqüência de oscilação do circuito $\omega$. Com estas considerações poderia-se esperar que para medir um ruído radiativo de destaque, é necessário medir as mais altas freqüências com um raio do solenóide maior. Porém isto não é verdade. Devemos lembrar de que os estudos sobre o ruído radiativo são não relativísticos (aproximação de comprimentos de onda longos), respeitando a 
condição (3.6). Portanto para medidas de freqüências grandes, o raio $a$ deve exatamente.

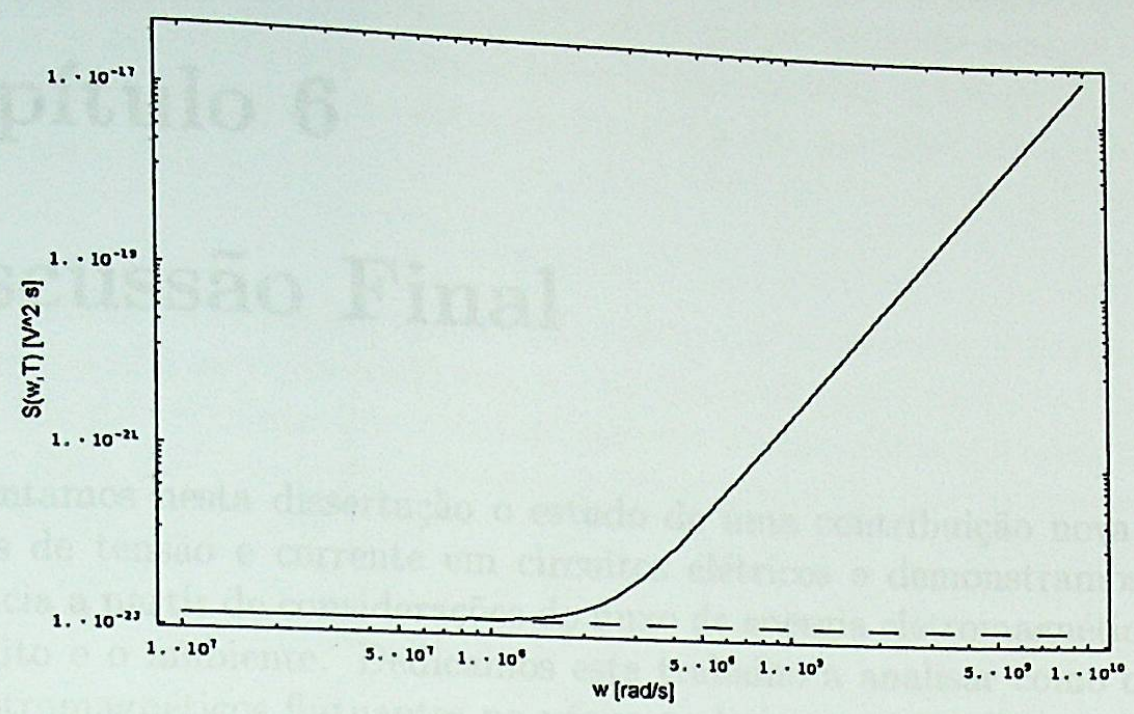

Figura 5.3: Gráfico das distribuições espectrais da tensão. A curva contínua representa o espectro total $S_{\varepsilon}(\omega, T)$ e a curva tracejada representa o espectro do ruído de Nyquist apenas $S_{N}(\omega, T)$. O solenóide é tal que $a=1 \mathrm{~cm}$, $N=1000$ e $R_{\text {ohm }}=1 \Omega$, a uma temperatura $T=3 \mathrm{~K}$.

Podemos ilustrar a relação entre o raio $a$ e as freqüências nas quais o ruído radiativo é relevante, comparando a figura $5.1 \mathrm{com}$ a figura 5.3. Na figura 5.3 é usado um solenóide com raio de $1 \mathrm{~cm}$ e com as outras características iguais ao caso da figura 5.1 , na qual o solenóide tem um raio de $0,1 \mathrm{~cm}$. O espectro $S_{\varepsilon}$ se destaca na figura 5.3 por volta da freqüência de $2 \cdot 10^{8} \mathrm{rad} / \mathrm{s}$, uma ordem de grandeza a menos do que na figura 5.1. 


\section{Capítulo 6}

\section{Discussão Final}

Apresentamos nesta dissertação o estudo de uma contribuição nova às flutuações de tensão e corrente em circuitos elétricos e demonstramos a sua existência a partir de considerações do fluxo de energia eletromagnética entre o circuito e o ambiente. Dedicamos este trabalho a analisar como os campos eletromagnéticos flutuantes no vácuo poderiam atuar sobre um circuito elétrico com dimensões físicas macroscópicas específicas. Assim foi feito um trabalho por Blanco, França, Santos e Sponchiado [4] predizendo a existência de flutuações de tensão devida a ação dos campos livres e relacionada com a resistência radiativa do circuito, tais flutuações no circuito foram chamadas de ruído radiativo. Foi tomado o caso particular em que o circuito é constituido basicamente de um solenóide macroscópico.

Nesse primeiro trabalho [4], apresentado na Seção 5.1, foi utilizada a idéia do teorema flutuação-dissipação para mostrar a possibilidade de existência do ruído radiativo. Assim foi mostrado que o ruído total no circuito é a simples soma do ruído de Nyquist, gerado pela resistência ôhmica do circuito, com o ruído radiativo, isto é,

$$
\begin{aligned}
S_{\varepsilon}(\omega, T) & =S_{N}(\omega, T)+S_{B}(\omega, T)= \\
& =\frac{\hbar \omega}{2 \pi}\left[R_{o h m}(\omega, T)+\frac{2 \pi^{2} N^{2}}{3 c}\left(\frac{a \omega}{c}\right)^{4}\right] \operatorname{coth}\left(\frac{\hbar \omega}{2 K T}\right) .
\end{aligned}
$$

A partir desse resultado, embora o ruído total seja diferente, mostrou-se que a energia média acumulada no circuito é igual à energia média acumulada apenas com o ruído de Nyquist.

Apesar do trabalho realizado por Blanco, França, Santos e Sponchiado [4] mostrar a possibilidade de existência do ruído radiativo, não mostrou que tal 
ruído deve necessariamente existir. Pois, mesmo que a densidade espectral da tensão flutuante seja alterada, não é possível observar qualquer mudança que, no equilíbrio termodina no circuito. Além disso, devemos considerar circuito e o ambiente a sua volt (os deve haver fluxo de energia entre o Isso significa que a média do vetor de campos eletromagnéticos do vácuo). deve ser nula, isto é,

$$
\langle\vec{S}\rangle=0 .
$$

Ora, se nenhuma radiação ou energia podem ser observadas a partir do circuito, então não se pode confirmar a existência do ruído radiativo. De fato, foi no cálculo explicito dos campos elétrico e magnético gerados pelo solenóide, feito por este autor, França e Sponchiado [5], que se comprovou a existência do ruído radiativo. No cálculo original feito em colaboração com o Prof. França e com Sponchiado [5], foi suposto que o solenóide está em equilíbrio com o ambiente a sua volta, de modo que a média do vetor de Poynting em qualquer ponto do espaço, para qualquer freqüência, deve ser nula. Considerando que os campos livres no vácuo agem sobre o solenóide, então o ruído radiativo deve necessariamente existir. Verificamos também que a densidade espectral dos campos elétrico e magnético resultantes em torno do solenóide devem ser diferentes do caso de campos eletromagnéticos no espaço livre. Contudo essa densidade espectral resultante é estável, isto é, ela não se altera ao longo do tempo e não se pode medir nenhuma radiação sendo absorvida ou emitida pelo solenóide.

As possibilidades de desenvolvimento da idéia do ruído radiativo são muitas. Visto que o ruído radiativo que estudamos é gerado por uma certa configuração específica do circuito, podemos esperar que o estudo de circuitos com diferentes configurações levarão a ruídos radiativos diferentes que podem levar a comportamentos qualitativamente diferentes. Naturalmente, podemos, por exemplo, procurar calcular as implicações que ocorrem, se o circuito tem um capacitor específico (placas planas paralelas, cilíndrico etc). Devemos mencionar que os cálculos relativos ao estudo da interação entre o solenóide e um dipolo elétrico microscópico, feitos por Blanco, Dechoum, França, Santos e Maia Jr. [1, 2, 3, 6] e apresentados no Capítulo 4, não são alterados com a inclusão do ruído radiativo no solenóide.

A teoria utilizada no estudo do ruído radiativo é a Eletrodinâmica Estocástica. O motivo do seu uso deve-se a seu sucesso obtido na descrição estatística dos campos eletromagnéticos livres, assim como no estudo do ruído 
de Nyquist. O ruído radiativo é um efeito oriundo da interação entre um
circuito composto de microscópicas do campo magnético macroscópico específico e as flutuações teoria que descreva tanto o mundo do vácuo. Assim, necessitamos de uma em vista o sucesso da Eletrodinâmicroscópico como o macroscópico. Tendo mas microscópicos relacionados com Estocástica em explicar vários sisteteoria clássica, o seu uso foi feito de tema desta dissertação, e sendo uma resultado novo muito plausível.

Após a obtenção de resultados teóricos, faz-se necessário as verificações experimentais. Propusemos a realização de experimentos capazes de observar flutuações do tipo do ruído radiativo. A possível confirmação da presença do dissertação, traria mais constos constituídos de solenóides como o proposto nesta Eletrodinâmica Estocástica. 


\section{Apêndice A}

\section{Campos de interação entre o solenóide e o dipolo elétrico}

\section{A.1 Campo elétrico gerado pelo solenóide}

Vamos calcular inicialmente o campo elétrico gerado pelo solenóide que age sobre o dipolo elétrico. Vamos considerar que o solenóide está disposto ao longo do eixo $0-z$ de $-l / 2$ a $l / 2$ e o seu centro está na origem de um sistema de coordenadas. Uma corrente flutuarte $I(t)$ percorre o solenóide, que é composto por $N$ espiras de raio $a$. Assim o momento de dipolo magnético gerado por $\frac{N}{l} d z$ espiras é

$$
d \vec{\mu}(t)=\frac{\pi a^{2} I(t)}{c}\left(\frac{N}{l} d z\right) \hat{z}
$$

dado que $\hat{z}$ o versor na direção $0-z$.

O potencial vetor $\vec{A}(\vec{r}, t)$ gerado pelo momento de dipolo magnético pode ser decomposto em uma parte espacial e outra temporal [16],

$$
\vec{A}(\vec{r}, t)=\vec{A}(\vec{r}) e^{-i \omega t},
$$

sendo que a parte $\vec{A}(\vec{r})$ é dada por [16]

$$
\vec{A}(\vec{r})=i k(\hat{n} \times \vec{\mu}) \frac{e^{i k r}}{r}\left(1-\frac{1}{i k r}\right) .
$$


Substituindo (A.1) em (A.2) e aplicando a transformada de Fourier em
ação ao tempo, temos

$$
d \vec{A}(\vec{r}, \omega)=\frac{\pi N a^{2}}{c l} \tilde{I}(\omega)(\hat{n} \times \hat{z}) e^{\frac{i \omega r}{c}}\left(\frac{i \omega}{c r}-\frac{1}{r^{2}}\right) d z .
$$

Como $\hat{n} \times \hat{z}=\hat{\phi}$, dado o versor polar $\hat{\phi}$, e $r^{2}=y^{2}+z^{2}$, então a expressão
(A.4) torna-se

$$
d \vec{A}(\vec{r}, \omega)=\frac{\pi N a^{2}}{c l} \tilde{I}(\omega) \sin (\theta) e^{\frac{i \omega}{c} \sqrt{z^{2}+y^{2}}}\left(\frac{i \omega}{c \sqrt{z^{2}+y^{2}}}-\frac{1}{z^{2}+y^{2}}\right) d z \hat{\phi},
$$

sendo que $\sin (\theta)=\frac{y}{\sqrt{z^{2}+y^{2}}}$, isto é, $\theta$ é o ângulo formado entre $\hat{n}$ e o eixo $0-z$.

A expressão (A.5) pode ser integrada com relação a variável $z$, assim

$$
\vec{A}(y, \omega)=\frac{\pi N a^{2} y}{c l} \tilde{I}(\omega) \int_{-\frac{l}{2}}^{\frac{l}{2}} d z e^{\frac{i \omega}{c} \sqrt{z^{2}+y^{2}}}\left[\frac{i \omega}{c\left(z^{2}+y^{2}\right)}-\frac{1}{\left(z^{2}+y^{2}\right)^{\frac{3}{2}}}\right] \hat{\phi}
$$

A transformada temporal inversa de Fourier do potencial vetor dado por (A.6) é

$$
\vec{A}(y, t)=\int_{-\infty}^{\infty} d \omega \vec{A}(y, \omega) e^{i \omega t}
$$

Desse modo é possivel escrever o campo elétrico gerado pelo solenóide sobre o eixo $0-y$, onde se encontra o dipolo elétrico,

$$
\vec{E}_{s o l}(t)=-\frac{1}{c} \frac{\partial \vec{A}}{\partial t}=\int_{-\infty}^{\infty} d \omega i \omega \tilde{I}(\omega) \frac{b(y, \omega)}{c^{2}} e^{i \omega t} \hat{\phi}
$$

sendo que

$$
b(y, \omega)=\frac{\pi N a^{2} y}{l} \int_{-\frac{l}{2}}^{\frac{l}{2}} d z e^{\frac{i \omega}{c} \sqrt{z^{2}+y^{2}}}\left[\frac{1}{\left(z^{2}+y^{2}\right)^{\frac{3}{2}}}-\frac{i \omega}{c\left(z^{2}+y^{2}\right)}\right] .
$$

A integral da função $b(y, \omega)$ pode ser feita, da qual se obtem

$$
b(y, \omega) \simeq \frac{\pi N a^{2} y}{l} \int_{-\frac{l}{2}}^{\frac{l}{2}} d z \frac{1}{\left(z^{2}+y^{2}\right)^{\frac{3}{2}}}=\frac{2 \pi N a^{2}}{y \sqrt{l^{2}+4 y^{2}}} .
$$


A seguir apresentamos o cálculo da tensão elétrica no solenóide induzida pelo do campo magnético produzido pelo $=y^{2}+z^{2}$, a componente na direção $0-z$

$$
\left(B_{d i p}\right)_{z}=\frac{q y \dot{x}(t-r / c)}{c r^{3}}+\frac{q y \ddot{x}(t-r / c)}{c^{2} r^{2}} .
$$
A tensão elétrica induzida no solenóide por este campo magnético é dada
pela lei de Faraday,

$$
\varepsilon_{\text {dip }}(t)=-\frac{1}{c} \frac{d \varphi}{d t}
$$

sendo que o fluxo magnético através do solenóide pode ser escrito como

$$
\varphi=\pi a^{2} \int_{-\frac{l}{2}}^{\frac{l}{2}} B_{z} \frac{N}{l} d z=\frac{\pi N a^{2}}{l} \int_{-\frac{l}{2}}^{\frac{l}{2}}\left[\frac{q y \dot{x}(t-r / c)}{c\left(z^{2}+y^{2}\right)^{\frac{3}{2}}}+\frac{q y \ddot{x}(t-r / c)}{c^{2}\left(z^{2}+y^{2}\right)}\right] d z .
$$

Aplicando a seguinte transformada de Fourier,

$$
x(t-r / c)=\int_{-\infty}^{\infty} d \omega \tilde{x}(\omega) e^{i \omega(t-r / c)}=\int_{-\infty}^{\infty} d \omega \tilde{x}(\omega) e^{i \omega t} e^{-i k r},
$$

nas funções $\dot{x}(t-r / c)$ e $\ddot{x}(t-r / c)$ da expressão (A.13), obtemos

$$
\varphi=\frac{\pi N a^{2} y}{c l} q \int_{-\infty}^{\infty} d \omega i \omega \tilde{x}(\omega) \int_{-\frac{l}{2}}^{\frac{l}{2}} d z e^{\frac{i \omega}{c} \sqrt{z^{2}+y^{2}}}\left[\frac{i \omega}{c\left(z^{2}+y^{2}\right)}-\frac{1}{\left(z^{2}+y^{2}\right)^{\frac{3}{2}}}\right] .
$$

Substituindo a expressão (A.15) na equação (A.12), temos

$$
\varepsilon_{d i p}(t)=-\frac{1}{c} \frac{d \varphi}{d t}=q \int_{-\infty}^{\infty} d \omega(i \omega)^{2} \tilde{x}(\omega) \frac{b(y, \omega)}{c^{2}} e^{i \omega t}
$$

sendo que $b(y, \omega)$ é dado pela expressão (A.10). 


\section{Referências Bibliográficas}

[1] R. Blanco, K. Dechoum, H. M. França and E. Santos, Phys. Rev. A $\underline{57}$
(1998) 724 .

[2] K. Dechoum, H. M. França and A. Maia Jr., Il Nuovo Cimento B $\underline{113}$
(1998) 455 .

[3] K. Dechoum, A Eletrodinâmica Estocástica e os Aspectos Clássicos da Teoria Quântica, Ph.D. Thesis, IFUSP, São Paulo (1998).

[4] R. Blanco, H. M. França, E. Santos and R. C. Sponchiado, Phys. Lett. A $\underline{282}$ (2001) 349.

[5] A. J. Faria, H. M. França and R. C. Sponchiado, Phys. Lett. A $\underline{345}$ (2002) 4.

[6] K. Dechoum and H. M. França, "Dynamical effects generated by the current fluctuations in a long solenoid" in: New Developments on Fundamental Problems in Quantum Physics, M. Ferrero and A. van der Merwe eds., Kluwer Academic, Dordrecht (1997).

[7] H. M. França and R. B. B. Santos, Phys. Lett. A $\underline{238}$ (1998) 227.

[8] H. M. França and R. B. B. Santos, Phys. Lett. A 251 (1999) 100.

[9] L. de la Peña and A. M. Cetto, The Quantum Dice. An Introduction to Stochastic Electrodynamics, A. van der Merwe ed., Kluwer Academic, Dordrecht (1996).

[10] A. Einstein and L. Hopf, Ann. d. Phys. $\underline{33}$ (1910) 1096. See also A. Einstein and L. Hopf, Ann. d. Phys. $\underline{33}$ (1910) 1105.

[11] T. H. Boyer, Phys. Rev. $\underline{182}$ (1969) 1374. 
[12] H. B. G. Casimir and D. Polder, Phys. Rev. $\underline{73}$ (1948) 360.

[13] T. H. Boyer, Phys. Rev. D 11 (1975) 790.

[14] T. H. Boyer, Phys. Rev. D 11 (1975) 809.

[15] P. W. Milonni, The Quantum Vacuum: An Introduction to Quantum Electrodynamics, Academic Press, Boston (1994), chapters 1, 2 and 4.

[16] J. D. Jackson, Classical Electrodynamics, 2nd ed., John Wiley and Sons, New York (1975), chapters 9 and 17

[17] W. Greiner and J. Reinhardt, Field Quantization, Springer-Verlag, Ber-
lin (1996), chapter 4 .

[18] N. G. van Kampen, Stochastic Processes in Physics and Chemistry, North-Holland, Amsterdam (1992), chapters 1 and 9.

[19] J. B. Johnson, Phys. Rev. $\underline{32}$ (1928) 97.

[20] H. Nyquist, Phys. Rev. $\underline{32}$ (1928) 110.

[21] H. B. Callen and T. E. Welton, Phys. Rev. 83 (1951) 34.

[22] H. B. Callen and R. F. Greene, Phys. Rev. 86 (1952) 702.

[23] V. L. Ginzburg, Applications of Electrodynamics in Theorical Physics and Astrophysics, Gordon and Breach, New York (1989), chapter 14.

[24] U. Weiss, Quantum Dissipative Systems, 2nd ed., World Scientific, London (1999), chapters 2 and 6, sections 3.1 and 4.4.

[25] R. Kubo, Rep. Prog. Phys. 29 (1966) 255.

[26] R. Kubo, M. Toda and N. Hashitsume, Statistical Physics II. Nonequilibrium Statistical Mechanics, 2nd ed., Springer-Verlag, Berlin (1998), chapters 1 to 4 .

[27] C. W. Gardiner, Quantum Noise, Springer-Verlag, Berlin (1991), chapter 3 .

[28] F. Reif, Statistical and Thermal Physics, McGraw-Hill, New York (1965), chapter 15. 
[29] L. D. Landau and E. M. Lifshitz, Statistical Physics, Pergamon Press,
London (1958), chapter 12.

[30] R. H. Koch, D. J. Van Harlingen and J. Clarke, Phys. Rev. Lett. 45
(1980) 2132. See also Phys. Rev. Lett. 47 (1981) 1216.

[31] H. M. França, T. W. Marshall and E. Santos, Phys. Rev. A $\underline{45}$ (1992)
6436 .

[32] W. Jhe, A. Anderson, E. A. Hinds, D. Meschede, L. Moi and S. Haroche,
Phys. Rev. Lett. 58 (1987) 666 . 